\title{
Optical dropout galaxies lensed by the cluster A2667 ${ }^{\star}$
}

\author{
N. Laporte ${ }^{1,2}$, R. Pellón ${ }^{1,2}$, D. Schaerer ${ }^{2,3}$, J. Richard ${ }^{4}$, E. Egami ${ }^{5}$, J. P. Kneib ${ }^{6}$, J. F. Le Borgne ${ }^{1,2}$, A. Maizy ${ }^{1,2}$, \\ F. Boone ${ }^{1,2}$, P. Hudelot ${ }^{7}$, and Y. Mellier ${ }^{7}$ \\ ${ }^{1}$ Université de Toulouse, UPS-OMP, IRAP, 31400 Toulouse, France \\ e-mail: [nicolas.laporte;roser]@ast.obs-mip.fr \\ 2 CNRS, IRAP, 14 Avenue Edouard Belin, 31400 Toulouse, France \\ e-mail: Daniel. Schaerer@unige.ch \\ 3 Geneva Observatory, 51 Ch. des Maillettes, 1290 Versoix, Switzerland \\ ${ }^{4}$ Institute for Computational Cosmology, Department of Physics, University of Durham, DH1 3LE, UK \\ e-mail: johan.richard@durham.ac.uk \\ 5 Steward Observatory, University of Arizona, 933 North Cherry Avenue, Tucson, AZ 85721, USA \\ e-mail: eegami@as.arizona.edu \\ ${ }^{6}$ Laboratoire d'Astrophysique de Marseille, CNRS - Université Aix-Marseille, 38 rue Frédéric Joliot-Curie, \\ 13388 Marseille Cedex 13, France \\ e-mail: jean-paul.kneib@oamp.fr \\ 7 Institut d'Astrophysique de Paris, UMR7095 CNRS, Université Pierre \& Marie Curie, 98 bis boulevard Arago, 75014 Paris, France \\ e-mail: [hudelot;mellier]@iap.fr
}

Received 30 September 2010 / Accepted 13 April 2011

\section{ABSTRACT}

Context. We investigate the nature and the physical properties of ten $z, Y$, and $J$-dropout galaxies selected in the field of the lensing cluster A2667.

Aims. This cluster is part of our project aimed at obtaining deep photometry at $\sim 0.8-2.5$ microns with ESO/VLT HAWK-I and FORS2 on a representative sample of lensing clusters extracted from our multi-wavelength combined surveys with Spitzer, HST, and Herschel. The goal is to identify a sample of redshift $z \sim 7-10$ candidates accessible to detailed spectroscopic studies.

Methods. Our selection is performed using the usual dropout technique based on deep $I, z, Y, J, H$, and $K$ s-band images (AB 26-27, $3 \sigma$ ), targeting $z \gtrsim 7.5$ galaxy candidates. We also include IRAC data between 3.6 and $8 \mu \mathrm{m}$, and MIPS $24 \mu \mathrm{m}$ when available. In this paper, we concentrate on the complete $Y$ and $J$-dropout sample among the sources detected with a high signal-to-noise ratio in both $H$ and $K$ s bands, as well as the bright $z$-dropout sources fulfilling the color and magnitude selection criteria adopted by Capak and collaborators. SED-fitting and photometric redshifts were used to constrain the nature and the properties of these candidates.

Results. Ten photometric candidates are selected within the $\sim 7^{\prime} \times 7^{\prime}$ HAWK-I field of view ( $33 \operatorname{arcmin}^{2}$ of effective area once corrected for contamination and lensing dilution at $z \sim 7-10$ ). All of these are detected in $H$ and $K$ s bands in addition to $J$ and/or IRAC $3.6 \mu \mathrm{m} / 4.5 \mu \mathrm{m}$ images, with $H_{A B}$ ranging from 23.4 to 25.2 , and have modest magnification factors between 1.1 and 1.4 . Although best-fit photometric redshifts are obtained at high- $z$ for all these candidates, the contamination by low- $z$ interlopers is expected to be in the range $\sim 50-75 \%$ based on previous studies, and on comparison with the blank-field WIRCAM Ultra-Deep Survey (WUDS). The same result is obtained when photometric redshifts are computed using a luminosity prior, allowing us to remove half of the original sample. Among the remaining galaxies, two additional sources could be identified as low- $z$ interlopers based on a detection at $24 \mu \mathrm{m}$ and the HST $z_{850}$ band. These low- $z$ interlopers are not accurately described by current spectral templates given the large break, and cannot be easily identified based on broad-band photometry in the optical and near-IR domains alone. A good fit at $z \sim 1.7-3$ is obtained when assuming a young stellar population together with a strong extinction. Given the estimated dust extinction and high SFRs, some of them could also be detected in the IR or sub-mm bands.

Conclusions. After correction for contaminants, the observed number counts at $z \gtrsim 7.5$ seem to agree with expectations for an evolving LF, and be inconsistent with a constant LF since $z \sim 4$. At least one and up to three candidates in this sample are expected to be genuine high- $z$ sources, although spectroscopy is still needed to confirm this.

Key words. gravitational lensing: strong - galaxies: high-redshift - dark ages, reionization, first stars

\section{Introduction}

Considerable advances have been made in the past few years in the exploration of the early Universe with the discovery of several $z \sim 6-7$ galaxies close to the end of the reionization epoch (e.g. Kneib et al. 2004; Stanway et al. 2004; Bouwens et al. 2004, 2008, 2009, 2010; Iye et al. 2006; Stark et al. 2007;

* Based on observations collected at the European Southern Observatory, Paranal, Chile, as part of the ESO 082.A-0163.
Bradley et al. 2008; Zheng et al. 2009), whereas five-year WMAP results place the first building blocks at $z=11.0 \pm 1.4$, suggesting that reionization was an extended process (Dunkley et al. 2009). For now, very few galaxies beyond $z \sim 6.5$ are spectroscopically confirmed (Hu et al. 2002; Cuby et al. 2003; Kodaira et al. 2003; Taniguchi et al. 2005; Iye et al. 2006), and the samples beyond this limit are mainly supported by photometric considerations (Kneib et al. 2004; Bouwens et al. 2004, 2006, 2008, 2009, 2010; Richard et al. 2006, 2008; Bradley et al. 2008; Zheng et al. 2009; Castellano et al. 2010; Capak et al. 2011). Strong evolution has 
been found in the abundance of galaxies between $z \sim 7-8$ and $z \sim 3-4$ (e.g. Bouwens et al. 2008) based on photometricallyselected samples, the star-formation rate (SFR) density being much lower at very high- $z$ up to the limits of the present surveys, in particular towards the bright end of the luminosity function (LF). A similar evolutionary trend is observed in narrow-band surveys searching for Lyman-alpha emitters (e.g. Iye et al. 2006; Cuby et al. 2007; Hibon et al. 2010).

Lensing clusters of galaxies provide a unique and priviledged view of the high-redshift Universe. This technique, often referred to as a "gravitational telescope", was first proposed by Zwicky (1937). It has proven to be highly successful in identifying several of the most distant galaxies known today thanks to magnifications of typically 1-3 mag (e.g. Ellis et al. 2001; Hu et al. 2002; Kneib et al. 2004; Bradley et al. 2008; Zheng et al. 2009).

Our project is based on the photometric pre-selection of high- $z$ candidates in lensing clusters using the Lyman-break technique (LBGs, e.g. Steidel et al. 1995), which has been proven to be successful in identifying star-forming objects up to $z \sim 6$ (Bunker et al. 2004; Bouwens et al. 2004-2009), as well as photometric redshifts. The long-term goal is to substantially increase the present sample of redshift $z \sim 7-12$ galaxies, and to study their physical properties and star-formation activity using a multi-wavelength approach.

This paper presents the first results of the short-term ongoing survey aimed at completing a deep photometric survey at $\sim 0.8-2.5$ microns with HAWK-I at ESO/VLT on a representative sample of strong-lensing clusters at intermediate redshift, extracted from our multi-wavelength combined survey with Spitzer/IRAC+MIPS, HST (ACS/WFC3/NIC3), Herschel OT key program, and sub-mm coverage. Herschel data of this field were obtained with PACS and SPIRE as part of the Herschel Lensing Survey (HLS, PI. Egami; see also Egami et al. 2010). These results and data will be described in a forthcoming paper. The presence of a strong lensing cluster within the large fieldof-view of HAWK-I $\left(\sim 7^{\prime} \times 7^{\prime}\right)$ is expected to optimize the global efficiency of the survey by combining in a single shot the benefit of gravitational magnification and a large effective surveyed volume (see also Maizy et al. 2010).

In this paper, we concentrate on the complete sample of $Y$ and $J$-dropout sources selected in the field of the lensing cluster A2667 (Abell 2667, $\alpha=23: 51: 39.35 \delta=-26: 05: 03.1$ $\mathrm{J} 2000, z=0.23$ ). For comparison, we also examine the bright $z$-dropout sources fulfilling the color and magnitude selection criteria adopted by Capak et al. (2011). All the candidates are selected to represent star-forming galaxies at $z \gtrsim 7.5$, and to be bright enough for spectroscopic follow-up, although a large fraction of galaxies selected in this way could be low- $z$ contaminants. SED-fitting and photometric redshifts are used to constrain the nature and the properties of these candidates. The results achieved on the luminosity functions in the $z \gtrsim 7.0$ redshift domain will be presented elsewhere.

In Sect. 2, we summarize the photometric observations and data reduction. We also describe the construction and analysis of photometric catalogs. The selection of different dropout candidates is presented in Sect. 3. The properties of the selected candidates, including spectral energy distributions (hereafter SEDs), SED-fitting results, and photometric redshifts are described in Sect. 4 , together with a discussion of the reliability of the different high- $z$ candidates when photometric redshifts include a luminosity prior. In Sect. 5, we discuss the global properties of this sample, in particular the expected versus observed number counts. We compare our results with previous findings and
Table 1. Photometric dataset: ground-based optical and near-IR imaging used in the selection of high- $z$ candidates (top) and space-based complementary data (bottom).

\begin{tabular}{lccccccc}
\hline \hline Filter & $\begin{array}{c}\lambda_{\text {eff }} \\
{[\mathrm{nm}]}\end{array}$ & $\begin{array}{c}C_{A B} \\
{[\mathrm{mag}]}\end{array}$ & $\begin{array}{c}t_{\text {exp }} \\
{[\mathrm{ks}]}\end{array}$ & $\begin{array}{c}\text { Pix } \\
{\left[{ }^{\prime \prime}\right]}\end{array}$ & $\begin{array}{c}m(3 \sigma) \\
{[\mathrm{mag}]}\end{array}$ & $\begin{array}{c}m(50 \%) \\
{[\mathrm{mag}]}\end{array}$ & $\begin{array}{c}\text { Seeing } \\
{\left[{ }^{\prime \prime}\right]}\end{array}$ \\
\hline$I$ & 793 & 0.45 & 13.0 & 0.126 & 27.5 & 26.8 & 0.47 \\
$z_{1}$ & 920 & 0.54 & 12.7 & 0.126 & 26.1 & 25.7 & 0.91 \\
$z_{2}$ & 920 & 0.54 & 13.2 & 0.126 & 26.0 & 25.7 & 0.54 \\
$Y$ & 1021 & 0.62 & 8.6 & 0.106 & 26.9 & 26.3 & 0.61 \\
$J$ & 1260 & 0.95 & 9.2 & 0.106 & 26.3 & 25.7 & 0.64 \\
$H$ & 1625 & 1.38 & 25.3 & 0.106 & 26.8 & 26.5 & 0.46 \\
$K \mathrm{~s}$ & 2152 & 1.86 & 11.0 & 0.106 & 25.9 & 25.8 & 0.47 \\
\hline & & & & & & Ref. & \\
$z 850$ & 910.6 & 0.54 & 8.8 & 0.05 & 26.1 & $\mathrm{~A}$ & \\
$3.6 \mu \mathrm{m}$ & 3575 & 2.79 & 16.8 & 1.2 & 25.1 & & \\
$4.5 \mu \mathrm{m}$ & 4528 & 3.25 & 17.4 & 1.2 & 25.2 & & \\
$5.8 \mu \mathrm{m}$ & 5693 & 3.70 & 2.4 & 1.2 & 22.7 & $\mathrm{~B}$ & \\
$8.0 \mu \mathrm{m}$ & 7958 & 4.37 & 2.4 & 1.2 & 22.8 & $\mathrm{~B}$ & \\
$24 \mu \mathrm{m}$ & 23843 & 6.69 & 2.7 & 2.55 & 18.7 & $\mathrm{C}$ & \\
\hline
\end{tabular}

Notes. Information given in this table: filter identification, filter effective wavelength, and $A B$ correction $\left(m_{A B}=m_{\text {Vega }}+C_{A B}\right)$, total exposure time, pixel size, $3 \sigma$ limiting magnitude, and average seeing. For the filters used in the high- $z$ sample selection, the $50 \%$ completeness level is also indicated (point source, 1.3" diameter aperture).

References. Richard et al. (2008), Egami et al. (2004), Rigby et al. (2008).

discuss the nature of low- $z$ contaminants. Conclusions are given in Sect. 6. The concordance cosmology is adopted throughout this paper, with $\Omega_{\Lambda}=0.7, \Omega_{\mathrm{m}}=0.3$ and $H_{0}=70 \mathrm{~km} \mathrm{~s}^{-1} \mathrm{Mpc}^{-1}$. All magnitudes are quoted in the $A B$ system (Oke \& Gunn 1983). Table 1 presents the conversion values between Vega and $A B$ systems for our photometric dataset.

\section{Observations and data analysis}

\subsection{Ground-based optical and near-IR imaging}

The selection of high- $z$ candidates is based on deep optical and near-IR imaging. A2667 was observed with HAWK-I in the near-infrared domain $(\sim 0.9$ to $2.2 \mu \mathrm{m}$, covering the four bands $Y, J, H$, and $K \mathrm{~s}$ ), and with FORS2 in $I$ and $z$ bands, between October and November 2008. Data reduction and processing included photometric calibration, bias subtraction, flat-fielding, sky subtraction, registration, and combination of images using IRAF, closely following the general procedures described in Richard et al. (2006). Table 1 summarizes the properties of the photometric dataset used in this paper.

For the FORS2 data, we used a standard flat-field correction and combination of the individual frames with bad-pixel rejection. In addition to the $z$ band image matching the HAWK-I field (hereafter $z_{1}$ ), an older image of similar quality centered on the cD galaxy, obtained in June 2003 (71.A-0428, hereafter $z_{2}$ ) was used to confirm the non-detection of high- $z$ candidates in the common area.

For HAWK-I data, we used the ESO pipeline ${ }^{1}$ to process and combine all individual images, refining the offsets between the different epochs of observations when producing the final stack. This procedure performs a two-step sky subtraction, using masks to reject pixels located on bright objects, similar

\footnotetext{
1 See http://www.eso.org/sci/data-processing/software/ pipelines/index.html
} 
to the XDIMSUM package described in Richard et al. (2006). The photometric calibration was checked against 2MASS stars present in the field of view, taking into account the relative flatfield normalisations between each one of the four HAWKI chips. Before combining, we applied individual weight values according to weight $\propto\left(Z P \times v a r \times s^{2}\right)^{-1}$, where individual zero-point $Z P$ and seeing $s$ values were obtained from the brightest unsaturated stars around the field, and pixel-to-pixel variance var was derived through statistics within a small region free of objects.

Photometric zero-points were derived from LCO/Palomar NICMOS standard stars (Persson et al. 1998). The final accuracy of our photometric calibration was checked by comparing the observed colors of cluster elliptical galaxies, measured on images matched to the worst seeing in our data (i.e. $\sim 0.9^{\prime \prime}$ in the $z_{1}$ band), to match expectations based on the empirical SED template compiled by Coleman, Wu, and Weedman (CWW 1980). According to this check, we expect our final photometric cata$\log$ to be accurate to about 0.05 mag throughout the wavelength domain between $I$ and $K \mathrm{~s}$.

All HAWK-I images were registered and matched to a common seeing using a simple Gaussian convolution to derive magnitudes and colors, the worst case being the $J$ band image. Astrometric calibration was performed in a standard way (see e.g. Richard et al. 2006), reaching an absolute accuracy of $\sim 0.2^{\prime \prime}$ for a whole HAWK-I field of view. Images in the $I$ and $z$ bands were registered to the HAWK-I images using standard IRAF procedures for rotation, magnification, and resampling of the data. These images were mainly used to exclude well-detected low- $z$ interlopers.

All high- $z$ candidates are expected to be detected in the $H$ and $K$ s bands and undetected in $I$ and $z$ bands. Therefore, the original $H$ and $K \mathrm{~s}$ band images were combined to create an $H+K$ s detection image with excellent seeing quality $\left(\sim 0.47^{\prime \prime}\right)$. The original unregistered images in $I$ and $z$ bands were used for the visual inspection of the $Y$-dropout candidates. Error bars and non-detection criteria were also determined for the original images (see below). Photometry for the $z$ and $Y$-dropout sample was also obtained with near-IR images matched to the worst seeing in our data to check for consistency with observed colors.

We used the SExtractor package version 2.8 (Bertin \& Arnouts 1996) for detection and photometry. Magnitudes were measured in all images with the SExtractor "double-image" mode, using the $H+K$ s detection image as a reference. Magnitudes were measured within different apertures ranging from 1 to $2^{\prime \prime}$ diameter, as well as MAG_AUTO magnitudes. We checked that colors derived with different choices of aperture and magnitude types are consistent with each other within the errors. Photometric errors were measured using the typical RMS in the pixel distribution of the original images (without any seeing matching or rescaling), within apertures of the same physical size as for flux measurements (either aperture or MAG_AUTO magnitudes). Photometric errors measured in the original images were also used to compute $3 \sigma$ limiting magnitudes in each band, which are reported in Table 1. Errors in the colors were derived from the corresponding magnitude errors added quadratically.

Completeness values for point-sources detected in the different bands were obtained using Monte Carlo techniques. Artificial stars (i.e., seeing limited sources) were added at $\sim 700$ different random locations on our images, then extracted using the same parameters for detection and photometry as for astronomical sources. The seeing was measured on the original co-added images. The corresponding results are shown in Fig. 1 and Table 1 .

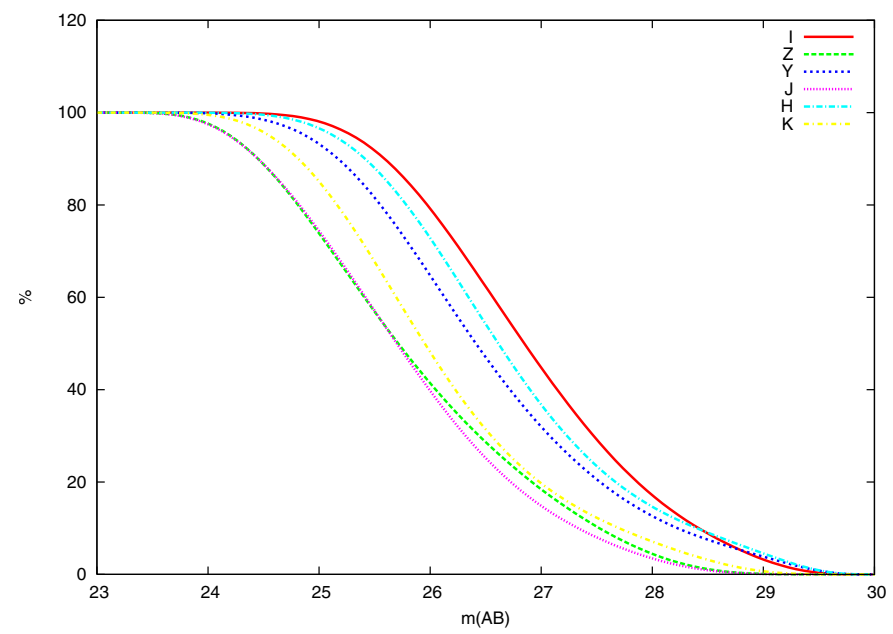

Fig. 1. Completeness levels obtained from simulations for the different bands used for high- $z$ sample selection: $I$ (solid red line), $z$ (dashed green line), $Y$ (dotted blue line), $J$ (dotted magenta line), $H$ (dot-dashed cyan line), and $K$ s (dot-dashed yellow line).

\subsection{Other imaging observations}

In addition to the images used for high- $z$ sample selection, SED analysis is also supported by additional data when available for the candidates, namely:

- images of Abell 2667 were obtained in the 3.6, 4.5, 5.8, and $8.0 \mu \mathrm{m}$ bands as part of the GTO Lensing Cluster Survey (Program 83, PI. Rieke), using the Infrared Array Camera (IRAC; Fazio et al. 2004) onboard the Spitzer Space Telescope (Werner et al. 2004). In addition, deeper exposures were obtained at 3.6 and $4.5 \mu \mathrm{m}$ in August 2009 (Program 60034, PI. Egami). All these images were processed according to Egami et al. (2006). IRAC magnitudes were measured within a $2^{\prime \prime}$ diameter aperture and corrected according to the Instrument Handbook (v.1.0, February 2010). The field of view is $5.2^{\prime} \times 5.2^{\prime}$;

- we also gathered $24 \mu \mathrm{m}$ images in this field obtained with MIPS (Rieke et al. 2004), which were processed as described by Rigby et al. (2008);

- a deep HST F850LP/ACS image is also available for the cluster core (8.8 ks, PI. Ellis; see also Richard et al. 2008). Photometry in this band is referred to as $z_{850}$ hereafter.

The properties of the complete photometric dataset used in this paper are summarized in Table 1, where the 3.6 and $4.5 \mu \mathrm{m}$ images refer to the most recent and higher quality dataset.

\section{Selection of high-z galaxy candidates}

The original catalog on the HAWK-I field of view includes $\sim 5 \times$ $10^{4}$ objects over a $\sim 45 \operatorname{arcmin}^{2}$ area with more than $75 \%$ of the exposure time on the HAWK-I images. Since high- $z$ candidates should be detected in both the $H$ and $K$ s bands, we first require a detection above $5 \sigma$ level in both filters within a $1.3^{\prime \prime}$ diameter aperture. This means selecting sources with $H<26.22$ and $K \mathrm{~s}<$ 25.43 , corresponding to a completeness level in our survey of $\gtrsim 70 \%$ for point sources (see Fig. 1). We also require less than a $2 \sigma$ level detection in both $I$ and $z$ bands. This selection yields a sample of 175 objects after removing all noisy areas on the $I$ and $z$ images, i.e. a common clean area of $\sim 42 \operatorname{arcmin}^{2}$ (equivalent to $\sim 33 \operatorname{arcmin}^{2}$ after correction for lensing dilution). 


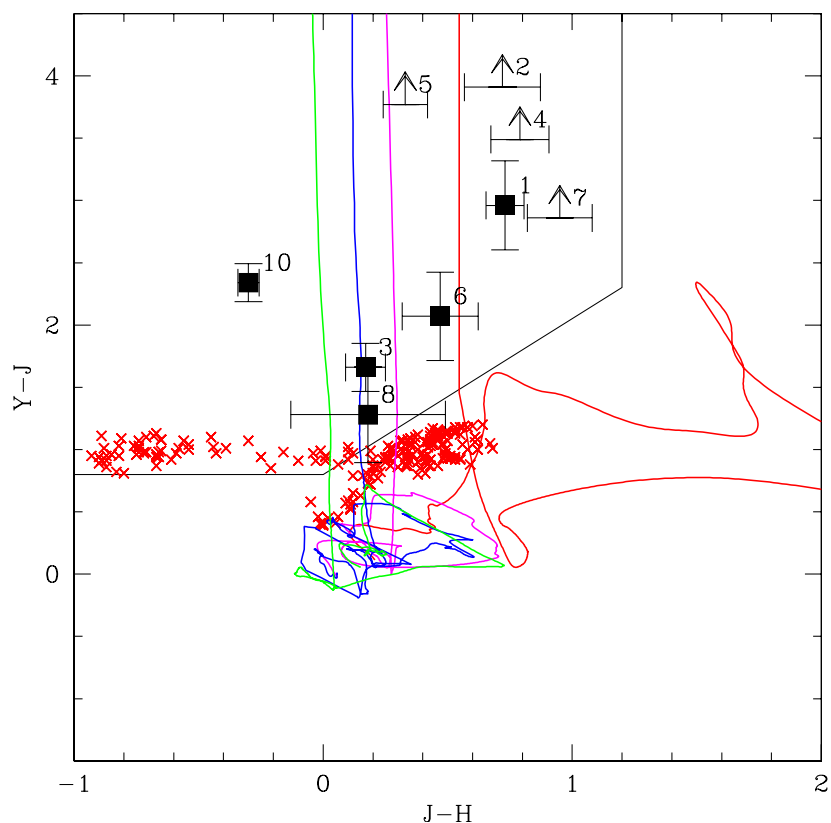

Fig. 2. Color-color diagram showing the position expected for spectral templates with redshifts $z \sim 0-9.0$ : E-type galaxies (CWW; red solid line), Im-type galaxies (blue line), and starburst templates of Kinney et al. (1996) (magenta and green lines). Red crosses show the colors of M, L, and T, stars from the SpeX Prism libraries of Burgasser et al. (2006). Black lines delimit our selection window for $z \gtrsim 7.3$ galaxies. Our $9 Y$-dropout candidates are marked with black squares and arrows: \#1 to \#8 correspond to $\mathrm{Y} 1$ to $\mathrm{Y} 8$, whereas \#10 is $\mathrm{z} 1$.

We then apply the following criteria to the remaining sample to select objects at $z>7$ :

(a) $Y-J>0.8, J-H<1.2$, and $Y-J>1.25 \times(J-H)+0.8$. This window, based on simulations using different spectral templates, selects $Y$-dropout $z \geq 7.3$ candidates. Figure 2 displays the corresponding color-color diagram for different models, namely E-type galaxies (CWW), Im-type galaxies (constant star-formation model from Bruzual \& Charlot 1993, 2003), and starburst templates of Kinney et al. (1996), together with the selection window. Other galaxy templates, such as the UV-to-radio spectral templates of galaxies and AGN from Polletta et al. (2007) used subsequently but not shown here, populate a very similar area in this and other color-color plots. We also show the synthetic colors of cool stars (M, L, T types) from the SpexGrism spectral library (see Burgasser et al. 2006, and references therein) ${ }^{2}$;

(b) $J-H>0.76, H-K \mathrm{~s}<0.5$, and $J-H>1.3 \times(H-K \mathrm{~s})+0.76$ illustrated in Fig. 3. Furthermore, we require a non-detection in the $Y$ band, below the $2 \sigma$ limit. This window is intended to select $J$-dropout candidates in the range $8 \lesssim z \lessgtr 10$ that are well detected in $H$ and $K$ s bands (cf. Richard et al. 2006). The corresponding color-color plot is shown in Fig. 3;

(c) The z-dropout selection criteria adopted by Capak et al. (2011) for their sample, i.e. $J<23.7$ (their $5 \sigma$ detection level), $z-J \geq 1.5, J-K \mathrm{~s}>0$, and $K \mathrm{~s}-4.5 \mu \mathrm{m}>0$ (cf. Fig. 4). The last criterium involving the $4.5 \mu \mathrm{m}$ band was not applied to our sample given the partial coverage of the HAWK-I field of view. Figure 4 displays the corresponding color-color plot. This selection does not in particular make use of the $Y$ band filter, which is in-between $z$ and $J$, available for our observations.

\footnotetext{
${ }^{2}$ See http://www. browndwarfs.org/spexprism
}

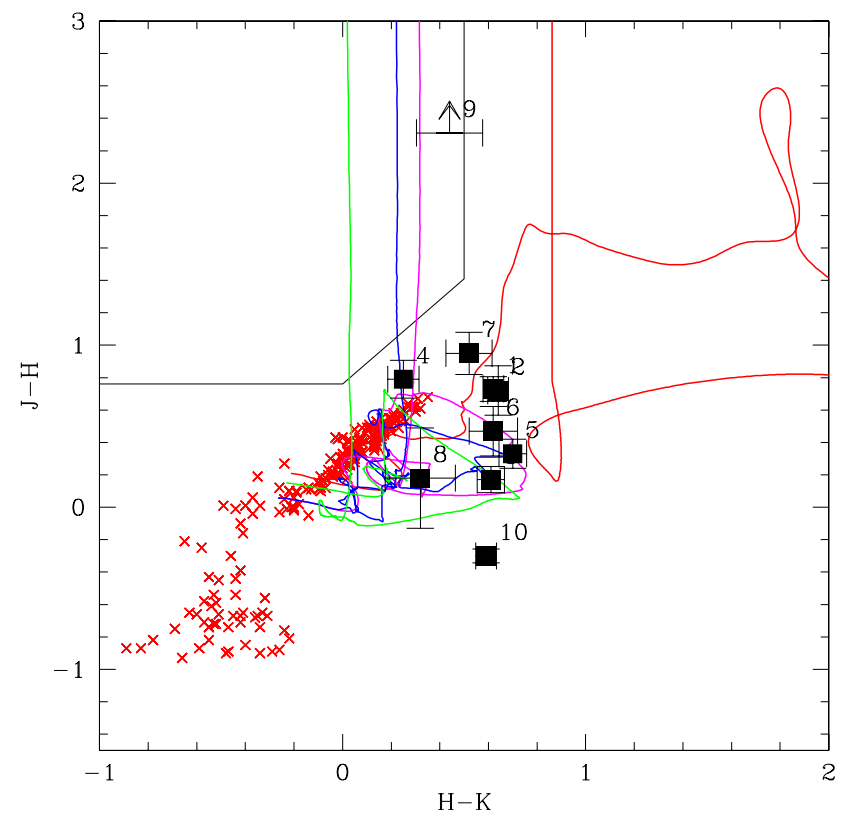

Fig. 3. Same as Fig. 2 for $(J-H)$ versus $(H-K)$ colors showing one $J$-drop candidate $(\mathrm{J} 1, \# 9)$ and the $9 Y$-dropout candidates (same identifications as in Fig. 2).

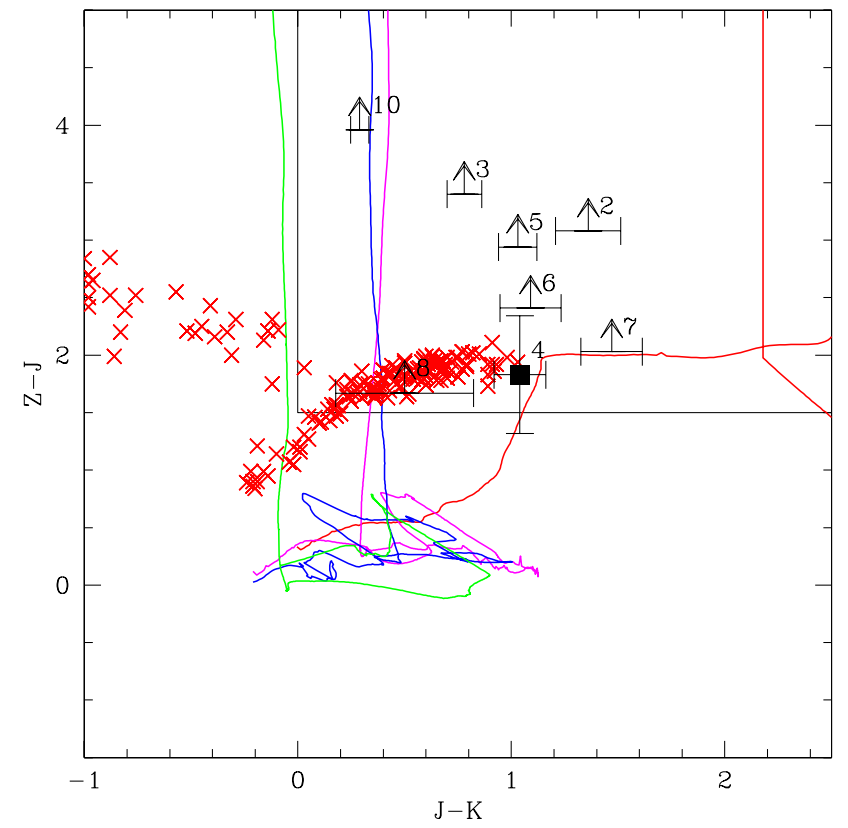

Fig. 4. Same as Fig. 4 for $(z-J)$ versus $(J-K)$ colors. Thin black lines denote the selection window (c) (the same as in Capak et al. 2011). The same identifications as in Figs. 2 and 3 are used for the dropout candidates.

There is some overlap between the $z$ and $Y$ bands, leading to somewhat ill-defined dropout criteria in $z-Y$. For this reason, we instead use $Y-J$ and $z-J$ in the above selection windows. Including the $Y$ band provides us with a useful discrimination tool between high- $z$ galaxies and cool stars, as shown in Fig. 2, while improving photometric redshifts.

The blind selection windows (a) and (b) include 48 and 52 sources, respectively ( 25 sources in common), i.e. 75 candidates in total out of the initial sample of 175 optical dropouts. We note that this selection does not introduce any restriction in magnitude, except for the $\geq 5 \sigma$ level detection in $H$ and $K \mathrm{~s}$. When 
Table 2. Catalogue of $z, Y$, and $J$-dropouts in A2667.

\begin{tabular}{|c|c|c|c|c|c|c|c|c|c|c|c|c|c|c|c|}
\hline Source & $\begin{array}{c}\text { RA } \\
(\mathrm{J} 2000)\end{array}$ & $\begin{array}{c}\text { Dec } \\
(\mathrm{J} 2000)\end{array}$ & $\bar{H}$ & $\Delta H$ & Stell. & $\begin{array}{c}F W H M \\
{\left[{ }^{\prime \prime}\right]}\end{array}$ & $\begin{array}{c}\mu \\
\text { high- } z\end{array}$ & $\begin{array}{c}\mu \\
\text { low- } z\end{array}$ & $\overline{\Delta \mu}$ & $Q 1$ & $Q 2$ & $Q 3$ & $\bar{Q}$ & $\chi_{\mathrm{opt}}^{2}$ & Final \\
\hline $\mathrm{z} 1$ & $23: 51: 45.837$ & $-26: 7: 07.20$ & 23.59 & 0.03 & 0.03 & 0.74 & 1.169 & 1.119 & 0.018 & 3 & 3 & 1 & $7-\mathrm{I}$ & -0.03 & (low) \\
\hline Y1 & 3:52:00.157 & $-26: 8: 30.31$ & 23.35 & 0.03 & 0.03 & 0.95 & 1.028 & 1.012 & 0.003 & 1 & 1 & 2 & 4-III & 1.00 & low \\
\hline Y2 & $: 51: 57.156$ & $-26: 4: 02.37$ & 23.45 & 0.03 & 0.40 & 0.58 & 1.108 & 1.031 & 0.007 & 2 & 2 & 1 & 5-III & 1.19 & low \\
\hline Y3 & $23: 51: 43.332$ & $-26: 8: 00.89$ & 23.68 & 0.04 & 0.02 & 1.24 & 1.128 & 1.059 & 0.012 & 3 & 3 & 2 & $8-\mathrm{I}$ & 0.54 & high \\
\hline Y4 & $23: 51: 35.750$ & $-26: 7: 10.65$ & 23.80 & 0.04 & 0.12 & 0.74 & 1.371 & 1.111 & 0.020 & 1 & 3 & 2 & 6-II & 0.64 & high \\
\hline Y5 & $3: 51: 54.448$ & $-26: 3: 13.65$ & 23.98 & 0.04 & 0.03 & 0.96 & 1.149 & 1.037 & 0.007 & 2 & 2 & 2 & 6-II & 0.68 & $?$ \\
\hline Y6 & $23: 51: 5$ & $-26: 4: 2$ & 24.37 & 0.08 & 0.02 & 1.81 & 1.167 & 1.067 & 0.011 & 1 & 1 & 3 & 5 -III & 0.07 & low \\
\hline Y7 & $23: 51: 56.568$ & $-26: 7: 51.45$ & 24.27 & 0.05 & 0.02 & 1.13 & 1.045 & 1.021 & 0.005 & 3 & 3 & 2 & $8-\mathrm{I}$ & 0.00 & low \\
\hline Y8 & $: 51: 37.151$ & $-26: 2: 30.46$ & 25.40 & 0.08 & 0.97 & 0.46 & 1.183 & 1.140 & 0.018 & 1 & 2 & 3 & 6-II & 0.58 & low \\
\hline $\mathrm{J} 1$ & 23:51:34.855 & $-26: 3: 32.74$ & 25.21 & 0.08 & 0.97 & 0.55 & 1.299 & 1.398 & 0.033 & 3 & 3 & 2 & $8-\mathrm{I}$ & 0.60 & (low) \\
\hline
\end{tabular}

Notes. Information given in this table: (1) Object identification; (2, 3) $\alpha$ and $\delta$ J2000; (4, 5) AUTO magnitudes and error bars in $H$ band; $(6,7)$ SExtractor stellarity index and maximum $F W H M$ measured on the detection $H+K$ s image; $(8,9,10)$ lensing magnification (high- $z$ and low- $z$ hypothesis) and associated uncertainty; $(11,12,13)$ quality grade for high- $z$ candidates according to three criteria: possible contamination by close neighbours $(Q 1)$, completeness and quality of photometric $\operatorname{SED}(Q 2)$, and UV luminosity $(Q 3)$; (14) total grade $Q ;(15)$ optical $\chi^{2}$, and (16) tentative classification between low and high- $z$ using a stringent prior in magnitude. In the case of $z 1$ and $\mathrm{J} 1$ (in brackets), the low- $z$ indentification is forced based on the detections in the $24 \mu \mathrm{m}$ and $z_{850}$ bands, respectively, for $\mathrm{z} 1$ and $\mathrm{J} 1$ (see Sect. 4.6).

applying the $z$-dropout selection criteria (c), 8 sources are found. All of these optical dropouts were carefully examined by a visual inspection in the different (original) bands to reject both spurious detections in the near-IR images and false non-detections in the $I$ and $z$ bands. The main sources of contamination were images detected within the haloes of bright galaxies leading to fake measurements or highly contaminated photometry. A mask was created to remove these regions from the subsequent analysis, that corresponds to $\sim 15 \%$ of the total surface.

We also checked that the selection based on the detection in $H$ and $K$ s bands does not introduce a bias against intrinsically blue sources (see e.g. Finkelstein et al. 2010). The selection was repeated with the requirement of a detection above $5 \sigma$ in the $H$ band alone, all the other conditions for optical dropouts being the same. This new selection includes all the previous objects plus four additional sources, but none of them survived the manual inspection.

At the end of the visual inspection, only 10 candidates survived from the original sample. Their photometry is listed in Table 3 . The brightest one in $J$ is the only source retained by selection criteria (c) after manual inspection (identified hereafter as z1). This source and 8 additional objects fulfill the $Y J H$ color selection (a) (cf. Fig. 2), which closely follows the selection previously adopted by Bouwens et al. (2008) and Richard et al. (2008) in $z J H$. The latter 8 objects, denoted as Y1 to Y8, are listed in order of increasing $H$ band magnitude within $1.3^{\prime \prime}$ aperture (cf. Table 3 ). We subsequently refer to these 9 sources as $Y$-drop candidates.

Only one candidate, J1, fulfills the selection criteria (b) based on $J H K \mathrm{~s}$, and is also consistent with the $z \sim 9 \mathrm{~J}$-drop selection criteria by Bouwens et al. (2010, see Fig. 3). This object was formally detected in the $I$ band at $\$ 2 \sigma$ level (double-image mode), as indicated in Table 3, although no convincing counterpart is seen in this image (see also Fig. 11). This source has a counterpart detected on the HST F850LP/ACS image, as discussed in Sect. 4.1 below.

We note that all $Y$-dropout candidates excepted $\mathrm{z} 1$ are too faint to be selected by the original Capak et al. criteria (i.e. intrinsic lensing-corrected $J \gtrsim 24$, see below). When the same color selection (c) is applied to fainter $J$-band magnitudes, up to $J<25.7$ (our own $5 \sigma$ detection level), 22 objects are selected after manual inspection. Among them, all our $Y$ candidates except Y4 are found. Indeed, Y4 was formally detected in the reference $z$ band at $\$ 2 \sigma$ level (as indicated in Table 3, double-image mode), although there is no clear counterpart seen on this image and it is also not detected in the $z_{2}$ field. This sample of fainter $z$-dropout candidates will be discussed elsewhere in a upcoming paper.

We concentrate hereafter on the bright $z$-dropout $\mathrm{zl}$ and the nine $Y$ and $J$-dropout candidates. For all these objects, we checked that their spectral energy distribution (hereafter SED) remains the same when using SExtractor aperture and MAG_AUTO magnitudes. MAG_AUTO magnitudes were preferred for the subsequent analysis because they are closer to total magnitudes.

\section{Results}

\subsection{Observed properties of the high-z candidates}

The identification, position, and morphology of the ten bright $Y$ and $J$-dropouts selected in this field is given in Table 2. Table 3 summarizes their photometric properties, and Fig. 11 displays the corresponding postage stamps. Except for J1, all candidates are detected in the $J, H$, and $K$ s bands. The observed $H$ and $K$ s band magnitudes of our objects range typically from $\sim 23$ to 25 , i.e. in a regime where our sample is close to $100 \%$ complete. For the high- $z$ candidates in the common area between $z_{1}$ and $z_{2}$ fields of view (namely z1, Y3, Y4, Y5, Y6, Y8 and J1), we also verified the independent non-detection in the two original images.

The SExtractor stellarity index ${ }^{3}$ and the $F W H M$ along the major axis, both measured on the $H+K$ s detection image, were used to quantify the morphology of our candidates. This information was only used, in addition to colors, to assess the possible contamination by cool stars. The reliability of the SExtractor stellarity index for galaxies diminishes towards the faintest magnitudes. We checked that a reliable index can be obtained up to $H K \sim 24.5$ for our combined image, becoming hazardous for fainter sources and highly unreliable at $H K \geq 25.0$, where the

\footnotetext{
3 This index ranges between 0.0 for extended sources and 1.0 for unresolved ones (see Bertin \& Arnouts 1996).
} 
Table 3. Photometric catalogue of bright $z, Y$, and $J$-dropouts in A2667.

\begin{tabular}{lccccccccccc}
\hline \hline Source & $I$ & $z$ & $Y$ & $J$ & $H$ & $K \mathrm{~s}$ & $3.6 \mu \mathrm{m}$ & $4.5 \mu \mathrm{m}$ & $5.8 \mu \mathrm{m}$ & $8.0 \mu \mathrm{m}$ & $24 \mu \mathrm{m}$ \\
& & & & & & & & & & & \\
\hline Z1(*) & $>28.7$ & $>27.2$ & 25.63 & 23.29 & 23.59 & 23.00 & 21.79 & 21.38 & - & 21.41 & 17.57 \\
& & & \pm 0.15 & \pm 0.03 & \pm 0.03 & \pm 0.03 & \pm 0.01 & \pm 0.01 & & \pm 0.10 & \pm 0.12 \\
Y1 & $>28.7$ & - & 27.04 & 24.08 & 23.35 & 22.73 & - & - & - & - & - \\
& & & \pm 0.35 & \pm 0.07 & \pm 0.03 & \pm 0.03 & & & & & - \\
Y2 & $>28.7$ & $>27.2$ & $>28.1$ & 24.17 & 23.45 & 22.81 & - & 21.80 & - & - & \\
& & & & \pm 0.15 & \pm 0.03 & \pm 0.03 & & \pm 0.01 & & & $>19.93$ \\
Y3(*) & $>28.7$ & $>27.2$ & 25.51 & 23.85 & 23.68 & 23.07 & 22.84 & 22.52 & - & - & \\
& & & \pm 0.18 & \pm 0.07 & \pm 0.04 & \pm 0.04 & \pm 0.04 & \pm 0.03 & & & \\
Y4(*) & $>28.7$ & 26.42 & $>28.1$ & 24.59 & 23.80 & 23.55 & 23.19 & 22.91 & $>23.93$ & $>23.98$ & $>19.93$ \\
& & \pm 0.50 & & \pm 0.11 & \pm 0.04 & \pm 0.05 & \pm 0.06 & \pm 0.04 & & & - \\
Y5(*) & $>28.7$ & $>27.2$ & $>28.1$ & 24.31 & 23.98 & 23.28 & 22.74 & 22.47 & - & - & - \\
& & & & \pm 0.08 & \pm 0.04 & \pm 0.04 & \pm 0.04 & \pm 0.03 & & & \\
Y6(*) & $>28.7$ & $>27.2$ & 26.91 & 24.84 & 24.37 & 23.75 & - & - & - & - & \\
& & & \pm 0.33 & \pm 0.13 & \pm 0.08 & \pm 0.06 & & & & & 18.78 \\
Y7 & $>28.7$ & $>27.2$ & $>28.1$ & 25.22 & 24.27 & 23.75 & 22.53 & 22.68 & - & - & \pm 0.36 \\
& & & & \pm 0.12 & \pm 0.05 & \pm 0.08 & \pm 0.03 & \pm 0.03 & & & \\
Y8(*) & $>28.7$ & $>27.2$ & 26.86 & 25.58 & 25.40 & 25.08 & - & - & 21.24 & 22.12 & $>19.93$ \\
& & & \pm 0.24 & \pm 0.30 & \pm 0.08 & \pm 0.12 & & & \pm 0.10 & \pm 0.19 & \\
J1(*) & 27.39 & $>27.2$ & $>28.1$ & $>27.5$ & 25.21 & 24.77 & 24.82 & 24.80 & 21.96 & 22.23 & $>19.93$ \\
& \pm 0.49 & & & & \pm 0.08 & \pm 0.11 & \pm 0.25 & \pm 0.22 & \pm 0.18 & \pm 0.21 & \\
\hline
\end{tabular}

Notes. Information given in this table: (1) object identification, AUTO magnitudes in filters $I$ to $K \mathrm{~s}$ (Cols. 2 to 7), corrected $2^{\prime \prime}$ diameter aperture magnitudes for IRAC 3.6, 4.5, 5.8 and $8.0 \mu \mathrm{m}(8,9,10,11)$, and MIPS $24 \mu \mathrm{m}(12)$. (*) These objects are also non-detected on the $13.2 \mathrm{ks} z$-band FORS2 image centered on the cluster core. Non-detections are indicated as $1 \sigma$ upper limits.

signal-to-noise ratio $(S / N)$ significantly drops below $\sim 10$. From Table 2, it appears that all our sources seem to be inconsistent with stars, except $\mathrm{Y} 8$ and $\mathrm{J} 1$, which are too faint for a robust morphological classification based on the detection image. As shown in Figs. 2 to 4, all candidates besides Y8 display colors that are clearly incompatible with cool stars.

$\mathrm{J} 1$ is the only candidate located on the central area covered by the HST F850LP/ACS image. A faint and compact object is indeed detected in this image, with FWHM $\sim 0.1^{\prime \prime}$ (SExtractor stellarity parameter is 0.7 ), and $z_{850}=27.39 \pm 0.18$ (MAG_AUTO on the original HST image). This magnitude is fully consistent with the non-detection in the ground-based $z$ band images $(A B \sim 27.2$ at $1 \sigma$ level $)$, and confirms the important break between optical and near-IR bands for this object, which is the faintest one in our sample.

High quality magnitudes were extracted for six and seven sources in the IRAC $3.6 \mu \mathrm{m}$ and $4.5 \mu \mathrm{m}$ images, respectively. For the remainder, only partial data is available (Y1, Y2), or the photometry is strongly contaminated by nearby sources (see Fig. 11). The only candidates clearly detected and reported in Table 3 for the shallow $5.8 \mu \mathrm{m}$ and $8.0 \mu \mathrm{m}$ images are $\mathrm{z} 1$, Y 8 and $\mathrm{J} 1$, even though magnitudes are dubious for $\mathrm{Y} 8$ and $\mathrm{J} 1$ because of a crowded environment. Two objects, $\mathrm{z} 1$ and $\mathrm{Y7}$, are clearly detected in the MIPS $24 \mu \mathrm{m}$ image.

\subsection{Magnification of the drop-out sources}

The lensing model for A2667 was originally obtained by Covone et al. (2006). We use this model to compute the magnification maps at different source redshifts with the public lensing software Lenstool ${ }^{4}$, including the new MCMC optimization method (Jullo et al. 2007) providing Bayesian estimates of model parameters.

\footnotetext{
${ }^{4}$ http://www. oamp.fr/cosmology/lenstool
}

The mass model was used to derive the magnification factor of each object and its associated error bars, for both the high and low- $z$ solutions (i.e. typical redshifts of $z \sim 7.5-9.0$ and $z \sim 1.7-2.0$ respectively, see Sect. 4.3). These values are given in Table 2 . The magnification factors of our objects typically range between 1.1 and 1.4, e.g. 0.4 mag being largest for Y4 and J1. None of these candidates is expected to be a multiple image.

Given the location of our candidates with respect to the critical lines, in either the high or in the low $-z$ solutions, the uncertainty in the magnification associated with the uncertainty in the redshift is in all cases smaller than $10 \%$. The magnification factor at a given position in the image plane varies slowly with redshift for sources located more than $\sim 10^{\prime \prime}$ apart from the critical lines, and this is indeed the case for all our candidates. Error bars in magnification are also given in Table 2, including both uncertainties in the source redshift and systematic errors due to the choice of the parametrization in lensing modelling (see e.g. Maizy et al. 2010). We also used this lensing model to compute the effective surveyed area and volume around $z \gtrsim 7.5$ when comparing with blank field surveys. All surface and volume number-densities given in this paper have been corrected for magnification by the lensing cluster.

\subsection{SED fitting results: photometric redshifts}

Photometric redshifts and associated probability distributions were derived for each of our candidates from the available broad-band photometry, except for the $24 \mu \mathrm{m}$ MIPS photometry, which is only available for a few candidates (see discussion below in Sect. 4.6). A modified version of the public photometric redshift software Hyperz (Bolzonella et al. 2000) was used, adapted to include nebular emission (see Schaerer \& de Barros 2009, 2010, hereafter SB2010). The following spectral templates were used for our SED fits: empirical templates (starbursts from Kinney et al. 1996; galaxies from Coleman et al. 1980; GRASIL templates from Silva et al. 1998; and the UV-to-radio 


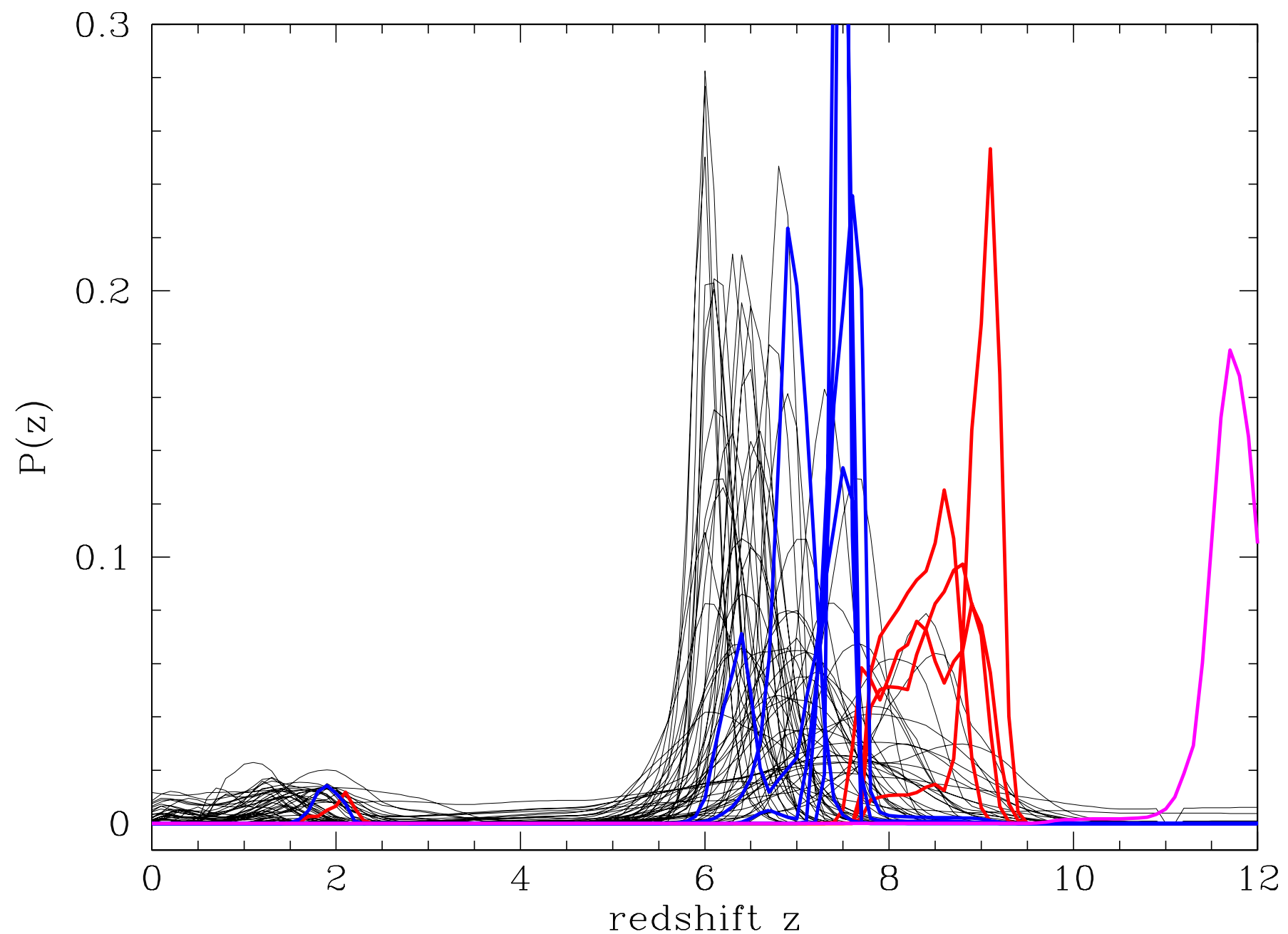

Fig. 5. Redshift probability distributions, $P(z)$ for our objects computed using standard templates (BC solar metallicity, no emission lines). Objects Y1, Y3, Y6, Y8, and z1 are shown in blue, Y2, Y4, Y5, and Y7 in red, and J1 in magenta. Black lines show $P(z)$ of other $z \sim 6-8$ galaxies from HST surveys using NICMOS and WFC3 from the analysis of Schaerer \& de Barros (2010), namely Gonzalez et al. (2010) and McLure et al. (2010) sample.

templates of galaxies and AGN from Polletta et al. 2007), and Bruzual \& Charlot (2003, hereafter BC) evolutionary sythesis models to which nebular emission (lines and continua) is added optionally.

The free parameters for the SED fits are the metallicity $Z$ (ranging from $\sim 1 / 50 Z_{\odot}$ to $Z_{\odot}$ ), the star-formation history, the age since the onset of star-formation, extinction, and redshift. For empirical templates redshift is in principle the only free parameter. However, in some cases we also allow for additional, variable extinction for empirical templates. Extintion is varied from $A_{\mathrm{V}}=0$ to 4 in steps of $0.2 \mathrm{mag}$, using the Calzetti et al. (2000) extinction law. The Lyman forest blanketing is included following the prescription of Madau (1995).

The non-detection in the $I, z, Y$, and $J$ bands was used as a constraint when computing photometric redshifts. Unless otherwise indicated, the rule applied corresponds to the usual case " 1 " of Hyperz, i.e. the flux in these filters is set to zero, with an error bar corresponding to the flux at $1 \sigma$ level, using both the global value and the local sensitivity computed near each source (see below). We also computed photometric redshifts by forcing the fluxes in these filters to be below the $2 \sigma$ and $3 \sigma$ levels using case " 2 " of Hyperz for non-detections. As explained below, the results obtained are rather insensitive to the non-detection rule and sensitivity applied (local or global). A minimum error bar of 0.1 mag was assumed for IRAC photometry to account for uncertainties in absolute flux calibrations when combining with the other filters.

The resulting redshift probability distributions $P(z)$ for all our objects, obtained with standard Bruzual \& Charlot, solar metallicity templates, is shown in Fig. 5. $P(z)$ displayed in this Figure were obtained using the Hyperz approach, i.e. $P(z) \propto$ $\exp \left(-\chi^{2}(z)\right)$, which is very similar to the results derived from Monte Carlo simulations. As shown by this figure, most objects have a relatively well-defined redshift probability distribution $P(z)$ peaking at high redshift. Five objects (Y1, Y3, Y6, Y8, and $\mathrm{z} 1$ ) have best-fit redshifts of $z_{\text {phot }} \sim 7-8$, four objects (Y2, Y4, Y5, and Y7) a higher redshift $z_{\text {phot }} \sim 7.5-9.5$, and for the $J$-drop J1 a value of $z_{\text {phot }}>9.5$ is the most likely one. These redshift ranges and their relative groupings are consistent with expectations on the basis of their colors (cf. Fig. 2). For most objects, a less significant solution is found at low- $z$, in general between $z \sim 1.7$ and 2.8. However, even though the high- $z$ solution produces a more accurate fit, several of these sources seem too bright $\left(M_{1500}<-23.0\right)$ to be at $z \geq 7.5$, suggesting some contamination by low- $z$ interlopers, which we discuss below.

Figure 5 also displays for comparison the $P(z)$ derived by SB2010 for a sample of $z \sim 6-8$ galaxies including objects from HST surveys using NICMOS and the latest WFC3 camera. The 
Table 4. Properties of bright $z, Y$, and $J$-dropouts in A2667.

\begin{tabular}{|c|c|c|c|c|c|c|c|c|c|c|c|c|c|c|c|c|}
\hline Source & $\begin{array}{c}z_{\text {phot }} \\
\text { high- } z\end{array}$ & $\chi^{2}$ & $z_{\min }-z_{\max }$ & $A_{\mathrm{V}}$ & $M_{1500}$ & $\begin{array}{c}L_{1500} \\
\times 10^{41} \\
\mathrm{erg} / \mathrm{s} / \mathrm{cm}^{2}\end{array}$ & $\begin{array}{c}S F R \\
M_{\odot} / \mathrm{yr}\end{array}$ & $\begin{array}{l}z_{\text {phot }} \\
\text { low-z. }\end{array}$ & $\chi^{2}$ & $A_{\mathrm{V}}$ & $M_{B}$ & $\begin{array}{l}P(z>6) \\
(1)\end{array}$ & (2) & (3) & (4) & \\
\hline z1 (a) & 7.6 & 75.08 & $7.5-7.7$ & 0.3 & -23.44 & 13.7 & 144 & 1.78 & 228.27 & 2.4 & -20.06 & 1.0 & 1.0 & 1.0 & 1.0 & (low) \\
\hline (b) & 7.6 & & & & & & & 1.94 & & & & & & & & \\
\hline Y1 (a) & 7.7 & 0.02 & $7.2-7.8$ & 2.4 & -23.12 & 10.2 & 108 & 1.72 & 39.62 & 0.3 & -19.82 & 1.0 & 0.0 & 1.0 & 1.0 & low \\
\hline (b) & 7.4 & & & & & & & 1.65 & & & & & & & & \\
\hline Y2 (a) & 8.7 & 0.89 & $7.7-9.3$ & 2.4 & -23.53 & 14.9 & 157 & 2.72 & 38.01 & 0.0 & -21.78 & 1.0 & 0.0 & 1.0 & 0.99 & low \\
\hline (b) & 9.1 & & & & & & & 2.11 & & & & & & & & \\
\hline Y3 (a) & 7.5 & 28.68 & $7.3-7.6$ & 1.2 & -22.97 & 8.9 & 94 & 1.88 & 72.94 & 0.6 & -20.32 & 1.0 & 0.97 & 1.0 & 0.79 & high \\
\hline (b) & 7.5 & & & & & & & 1.95 & & & & & & & & \\
\hline Y4 (a) & 9.1 & 6.98 & $8.7-9.4$ & 1.2 & -23.14 & 10.4 & 110 & 2.58 & 73.71 & 0.0 & -21.13 & 1.0 & 1.0 & 1.0 & 0.99 & high \\
\hline (b) & 9.2 & & & & & & & 2.11 & & & & & & & & \\
\hline Y5 (a) & 8.6 & 10.82 & $7.7-8.8$ & 2.1 & -22.97 & 8.9 & 94 & 1.70 & 40.95 & 0.0 & -19.32 & 1.0 & 0.63 & 1.0 & 0.98 & $?$ \\
\hline (b) & 8.3 & & & & & & & 1.94 & & & & & & & & \\
\hline Y6 (a) & 7.5 & 0.14 & $6.6-7.7$ & 1.2 & -22.15 & 4.2 & 44 & 1.94 & 3.64 & 1.50 & -19.74 & 0.94 & 0.0 & 0.55 & 0.46 & low \\
\hline (b) & 7.5 & & & & & & & 1.87 & & & & & & & & \\
\hline Y7 (a) & 9.1 & 0.05 & $8.0-9.4$ & 1.8 & -22.90 & 8.3 & 87 & 1.72 & 10.5 & 0.3 & -18.87 & 0.99 & 0.0 & 0.93 & 0.41 & low \\
\hline (b) & 9.2 & & & & & & & 2.11 & & & & & & & & \\
\hline Y8 (a) & 7.4 & 0.02 & $5.9-7.7$ & 0.3 & -21.29 & 1.9 & 20 & 1.66 & 0.77 & 0.6 & -18.33 & 0.82 & 0.05 & 0.37 & 0.26 & low \\
\hline (b) & 7.4 & & & & & & & 1.70 & & & & & & & & \\
\hline J1 (a) & 11.9 & 4.93 & $9.6-12.0$ & 0.0 & -22.66 & 6.7 & 71 & 2.80 & 12.48 & 0.0 & -20.19 & 1.0 & 0.94 & 0.85 & 0.20 & (low) \\
\hline (b)(c) & 11.8 & & & & & & & 2.50 & & & & & & & & \\
\hline
\end{tabular}

Notes. Information given in this table: (Col. 1) object identification; $(2,3,4,5,6,7,8)$ best-fit photometric redshift at high- $z, \chi^{2}, 1 \sigma$ confidence interval, best fit $A_{\mathrm{V}}$, magnification corrected $M_{1500}, L_{1500}$ and SFR from Kennicutt (1998) calibration; $(9,10,11,12)$ best-fit photometric redshift at low- $z$ and corresponding $\chi^{2}$, best fit $A_{\mathrm{V}}$ and $M_{B} ;(13,14,15,16)$ integrated probability distribution for $z>6$, normalized to 1 , for different cases based on Bruzual \& Charlot models: (1) non-detection rule "1" of Hyperz, (2) the same for a $P(z)$ including a luminosity prior with non-detection rule " 2 " of Hyperz (where the flux and the error bars are set to $F_{\lim } / 2$ ), and with $F_{\lim }=2 \sigma(3)$ or $3 \sigma$ (4) detection level in all filters where the candidates are formally not-detected; (17) tentative classification between low and high- $z$ using a luminosity prior. In the case of z1 and J1 (in brackets), the low- $z$ indentification is forced based on the detections in the $24 \mu \mathrm{m}$ and $z_{850}$ bands respectively for z1 and J1 (see Sect. 4.6 and Table 2). (a) Standard Hyperz models (local non-detection limits). (b) Complete library including nebular emission (global non-detection limits). (c) Photometric redshift for this source includes the detection in the $z_{850}$-band.

corresponding $P(z)$ of our candidates is comparable to and overlaps with these samples, and our selection function has clearly favoured the $z>7$ domain.

Using standard spectral templates (neglecting the effects of nebular emission), we obtain the best-fit photometric redshifts and physical parameters given in Table 4 . We also examined how the inclusion of nebular lines and continuous emission may alter the photometric redshift. In Fig. 6 and Table 4 we show the bestfit SEDs for all our objects in two different cases of without any redshift prior, and with the restriction of $z<4$. In all cases, the best-fit solution is found at high $z$, irrespective of the inclusion or not of nebular emission. Low redshift solutions (typically at $z \sim 1.7-2.1$ and $z \sim 2.5-2.8$ for J1) provide fits of lower quality, especially close to the spectral break and between $Y, J$, and $H$, as could be expected from the behaviour of spectral templates in these colours. Furthermore, these best-fit, low-redshift solutions generally show excess in the optical bands $(I, z)$ indicating that most of these objects should be detected at a 3-5 $\sigma$ level in at least one of these bands. We note that in some cases the inclusion of nebular emission allows for somewhat "unexpected" solutions with strong emission lines and a high attenuation (see e.g. the fits for $\mathrm{z} 1, \mathrm{Y} 8$, and $\mathrm{J} 1$ ), although the resulting low- $z$ fits remain with a higher $\chi^{2}$.

We investigated the influence of non-detection rules and limiting fluxes on photometric redshifts results. When applying a local non-detection limit instead of the global one, there is no difference in the high- $z$ solutions ( similar $\chi^{2}$ and $\Delta z<0.1$ ). Low redshift fits display the same lower quality as those at to high- $z$, with similar $\chi^{2}$ and, in general, $\Delta z \sim 0.1$, although there are larger differences for Y2 $(\Delta z \sim 1)$, Y4 $(\Delta z \sim 0.4)$, and Y5
$(\Delta z \sim 0.2)$. As shown in Table 4 , the same differences in $\Delta z$ are observed when comparing the photometric redshifts achieved with local non-detections and the standard Hyperz models, with those found with global non-detections and the complete library (including nebular emission). In other words, the dispersion in photometric redshifts between local and global non-detection limits is similar to the dispersion caused by model uncertainties. And, in all cases, the high- $z$ solution is the most likely one.

We also computed photometric redshifts by replacing the non-detection rule " 1 " of Hyperz by rule " 2 " in all filters where the candidates are formally not-detected, where the flux and the error bars are set to $F_{\lim } / 2$, for two different cases, $F_{\text {lim }}=2 \sigma$ and $3 \sigma$ detection levels. Best-fit redshifts remain precisely the same for most of our candidates at the $2 \sigma$ level, the only exception being Y8 (degenerate solution with best fit at $z=1.7$ ). When the fluxes are allowed to reach a $3 \sigma$ level, three other objects become degenerate, by having an additional best-fit at low$z$, namely Y6, Y7, and J1. Table 4 summarizes the integrated probability distribution at $z>6$ for all candidates when using different assumptions for non-detections.

As mentioned above, there is some overlap between the $z$ and $Y$ bands, but the $Y$-band allows us to improve our photometric redshifts. Indeed, when SED fitting results are derived without $Y$-band data, we still obtain a best-fit at high- $z$ for all objects. The $P(z)$ distribution for all objects detected in $Y$ (blue lines in Fig. 5) becomes broader (from $z_{\text {phot }}$ well peaked at 7-8 to $\left.z_{\text {phot }} \sim 6.5-9\right)$. For all other objects the changes in $P(z)$ are minor. When both $Y$ and $J$-band data are removed, this strongly degrades the photometric redshift $P(z)$ distribution and $z_{\text {phot }}$ basically becomes undefined for all objects. 
N. Laporte et al.: Optical dropout galaxies lensed by the cluster A2667
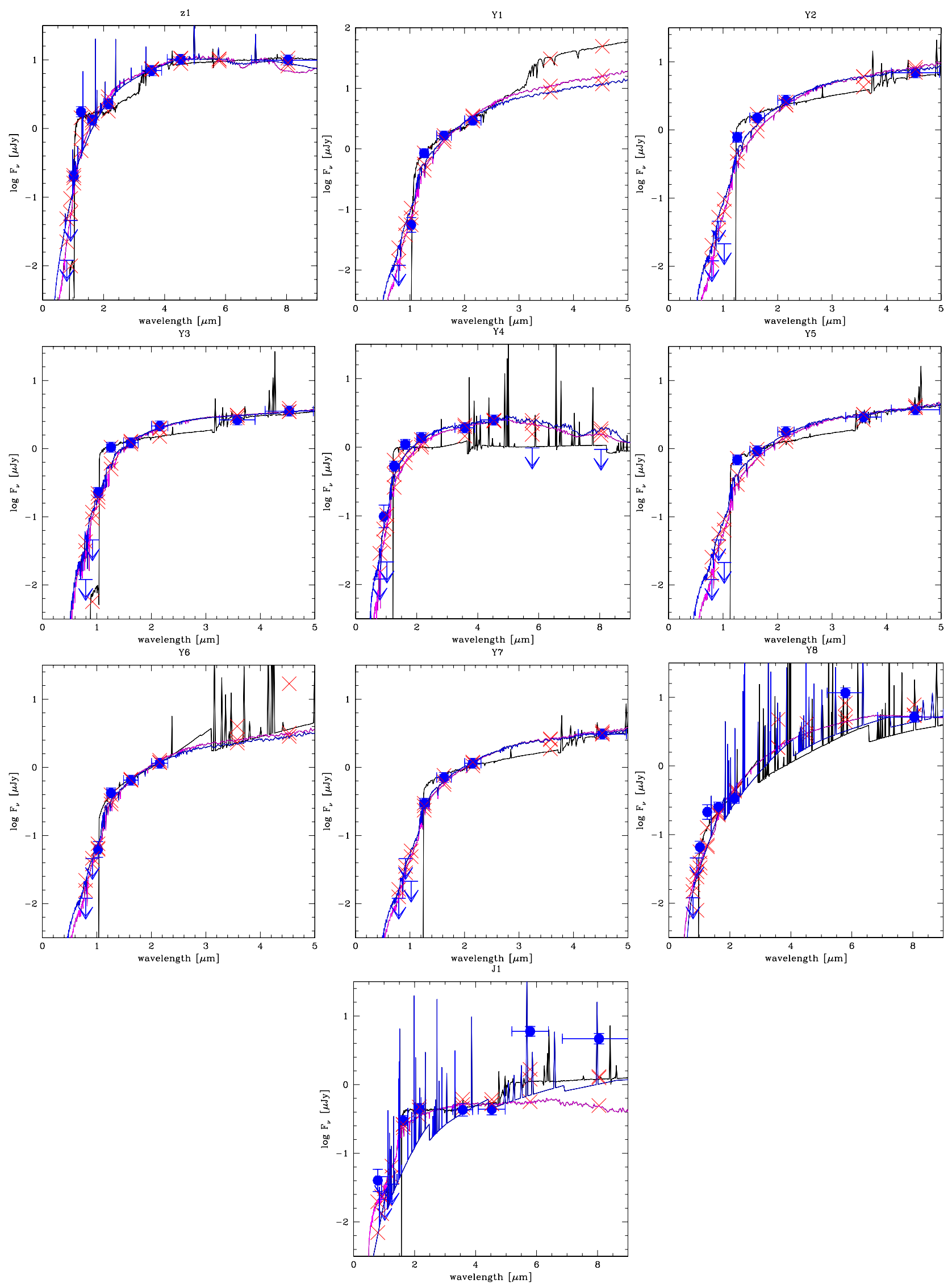

Fig. 6. Best-fit SED solutions for high- $z$ (black lines) et low- $z$ (blue and magenta lines) for all the bright $z, Y$, and $J$-dropouts found in A2667. Error bars and upper limits correspond to $1 \sigma$ values, as reported in Table 3. Red crosses indicate the synthetic flux in the filters. High- $z$ (black) spectra include nebular emission. Low- $z$ solutions are displayed for the complete library (including nebular emission) in blue, and for the standard templates in magenta. Note the extended wavelength scale for objects with available 5.8 and $8.0 \mu \mathrm{m}$ photometry. 


\subsection{Quality grades}

Table 2 includes two different quality grades for each source representing its likelihood of being a genuine high- $z$ candidate. The first one is based on the quality of the photometric information gathered for the source in terms of surrounding environment, completeness of the SED, and intrinsic UV luminosity if at high$z$. The second grade is based on the robustness of the optical non-detection criteria, following Bouwens et al. (2010).

The first grade $Q$ includes three independent criteria introduced as follows:

- the first one $(Q 1)$ is the quality of the surrounding environment, representing the possible contamination by neighbouring or underlying sources. Although all candidates are isolated in the good-seeing detection image $H+K \mathrm{~s}$, the presence of another source within a distance of $2^{\prime \prime}$ is given the lowest grade $(=1)$, whereas isolated candidates without neighbours closer than $\geq 3^{\prime \prime}$ have the highest grade $(=3)$;

- the second one $(Q 2)$ is the quality of the photometric SED. Objects with robusts constraints available beyond the $K \mathrm{~s}$ band, including 5.8 and $8.0 \mu \mathrm{m}$ bands, are given the hightest grade $(=3)$. The lowest grade $(=1)$ is given to sources lacking one or several bands at $\lambda \leq 4.5 \mu \mathrm{m}$, either because of field coverage or because of confusion problems;

- the third one $(Q 3)$ is the UV luminosity of the candidate at the best-fit photometric redshift, after correction for lensing effects. The hightest $(=3)$ and the lowest $(=1)$ grades are given, respectively, to sources fainter than $3 L^{*}$ and brighter than $\sim 10 L^{*}$, where $L^{*}$ stands for the Reddy \& Steidel (2009) value assuming no evolution.

As a result of these criteria, the most likely sources are given the highest cumulated value of $Q=Q 1+Q 2+Q 3$, allowing us to define a final grade that represents the quality of a given candidate, ranging between 3 and 9 (for an ideal candidate). As seen in Table 2, four candidates achieve the highest rates, between $Q=7(\mathrm{z} 1)$ and $Q=8(\mathrm{Y} 3, \mathrm{Y} 7$, and $\mathrm{J} 1)$. In all these cases, the high intrinsic luminosity is responsible for a lower value of Q3 with respect to the ideal case. These sources are considered as highest quality or category I. Three candidates achieve a fair value of $Q=6$ (Y4, Y5 and Y8) and are therefore considered as reasonably good (category II) candidates. The lowest grade (category III) is achieved for sources with close neighbours potentially affecting the quality of the global SED (Y1, Y2, and Y6).

The second grade is given by the optical $\chi_{\mathrm{opt}}^{2}$ computed on the optical bands as (see also Bouwens et al. 2010)

$\chi_{\mathrm{opt}}^{2}=\sum_{i} S G N\left(f_{i}\right)\left(\frac{f_{i}}{\sigma_{i}}\right)^{2}$,

where $f_{i}$ is the flux in the band $i, \sigma_{i}$ is the corresponding uncertainty, and $S G N\left(f_{i}\right)$ is equal to 1 if $f_{i}>0$ and equal to -1 if $f_{i}<0$. We used the IRAF package qphot.apphot to measure fluxes in a $1.3^{\prime \prime}$ diameter aperture, together with the corresponding noise in the neighbouring sky region. The $\chi_{\mathrm{opt}}^{2}$ values reported in Table 2 are based on $I$ and $z$-band images. In the case of $\mathrm{J} 1$, the $Y$-band was also included in the $\chi_{\mathrm{opt}}^{2}$ calculation. Straightforward simulations were conducted to determine the $\chi_{\text {opt }}^{2}$ distribution expected for truly non-detected sources as well as for sources at different $S / N$ values, in particular for those close to the $2 \sigma$ non-detection criteria in $I$ and $z$. All genuine nondetected sources exhibit $\chi_{\mathrm{opt}}^{2}<2$, with $90 \%$ at $\chi_{\mathrm{opt}}^{2}<1$ level, whereas only $3 \%$ of sources with $S / N \sim 2 \sigma$ in $I$ and $z$ are found with $\chi_{\mathrm{opt}}^{2}<2\left(1 \%\right.$ with $\left.\chi_{\mathrm{opt}}^{2}<1\right)$. As seen in Table 2 , all our

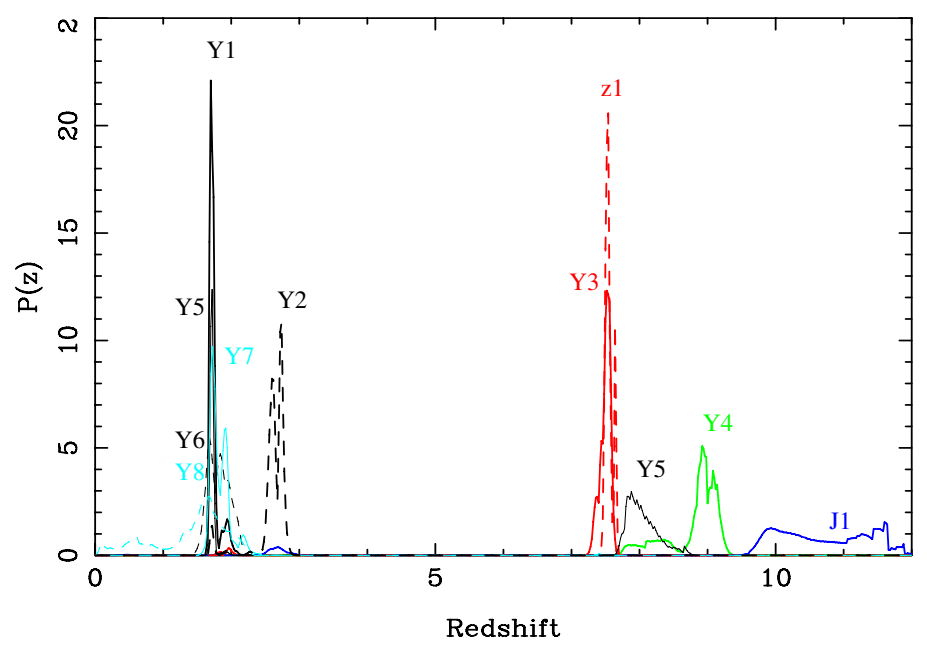

Fig. 7. Redshift probability distributions for the sample of high- $z$ candidates, arbitrarily normalized to 100 between $z=0$ and 12 , resulting from Hyperz $P(z)$ multiplied by a smooth luminosity prior.

candidates exhibit $\chi_{\text {opt }}^{2} \lessgtr 1$, the highest values corresponding to Y1 and Y2 (already ranked among the category III above).

These two quality grades above provide a useful priority for spectroscopic follow-up, although they do not take into account all the details regarding SED-fit constraints, as discussed below, which are somehow model-dependent. In particular, the $24 \mu \mathrm{m}$ emission, which is difficult to reconcile with a high- $z$ identification for $\mathrm{z} 1$ and $\mathrm{J} 1$ despite a high grade. The detection of $\mathrm{J} 1$ in the HST $z_{850}$-band is consistent with a low- $z$ solution for this source, which is considered hereafter as a possible interloper.

\subsection{Photometric redshifts with a luminosity prior}

Given the luminosities derived for these candidates in the high- $z$ hypothesis, leading to rather extreme masses and star-formation rates, it seems likely that, despite a best-fit solution existing at high- $z$, a large fraction of them actually corresponds to low- $z$ interlopers. To quantify this contamination more accurately, we introduced a luminosity prior when computing photometric redshifts.

A prior probability distribution was introduced as a function of redshift and magnitude, following Benitez (2000). In this case, the prior probability is the redshift distribution for galaxies of a given apparent magnitude $m$. Given the wide redshift domain covered by the $P(z)$, and that we are likely dealing with either genuine high- $z$ or $z \sim 1.5-2.5$ star-forming galaxies, we computed the prior probability based on the luminosity function for star-forming galaxies in the $B$-band (Ilbert et al. 2005). This band is indeed directly "seen" by the SED of galaxies in our sample for all redshifts between $z \sim 0.8$ and 9. A smooth probability distribution prior was computed for each object as a function of redshift, with the absolute magnitude $M_{B}$ derived from the apparent magnitude $m$, which is closer to the rest-frame $B$ band. The final probability distribution is given by the previous Hyperz $P(z)$ multiplied by the prior.

Figure 7 displays the resulting probability distributions for all candidates, arbitrarily normalized to 100 between $z=0$ and 12. As seen in this figure, four candidates still exhibit a dominant high- $z$ solution, namely z1, Y3, Y4, and J1, whereas one candidate is degenerate between low and high-z (Y5). The most likely high- $z$ candidates also exhibit the highest quality grades, 


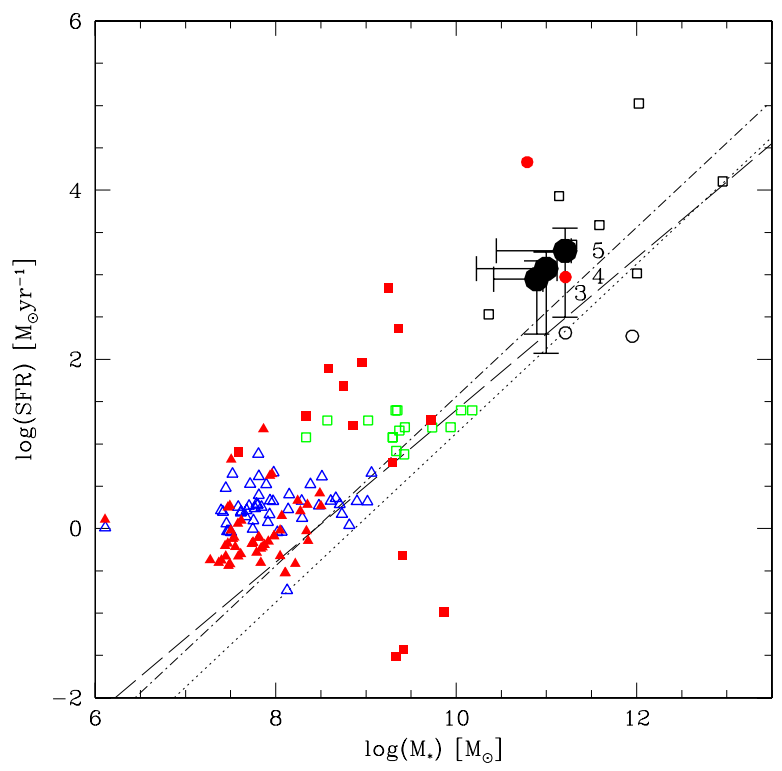

Fig. 8. Mass-SFR relation of the $z, Y$, and $J$-dropout galaxies resulting from $10^{3}$ Monte Carlo simulations compared to the properties of $z \sim 6-8$ galaxies from surveys analysed by Schaerer \& de Barros (2010). The most likely high- $z$ candidates based on photometric redshifts with a luminosity prior, namely $\mathrm{Y} 3, \mathrm{Y} 4$, and $\mathrm{Y} 5$, are identified and displayed by large black dots. The positions derived for the other optical-dropouts in this study, if at high- $z$, are indicated by black open squares for comparison. Open symbols (squares, triangles) show the "standard" SFR(UV) value (not corrected for extinction) versus mass derived from the SB2010 reference model for objects from their bright, intermediate, and faint samples, respectively. Red filled symbols show the best-fit model SFR and $M_{\star}$ values when assuming $\tau \geq 10 \mathrm{Myr}$ (model 1 in SB2010). Circles correspond to the two objects from Capak et al. (2011). The dotted (dash-dotted) lines show the locus for $S F R=$ const. from $z=\infty$ (10) to 7 corresponding to $S S F R=1.3$ (3.6) $\mathrm{Gyr}^{-1}$. The dashed line shows the relation found by Daddi et al. (2007) for $z \sim 2$ star-forming galaxies. The large spread in SFR is in particular due to a wide variety of exponentially decreasing star-formation histories beeing allowed. Note that if at high redshift the properties of our galaxies follow the trends observed for less massive/bright objects a high redshift.

as seen in the previous section. We use these results to propose a final tentative classification between likely low- $z$ interlopers and high- $z$ candidates in Table 2. Despite a high grade, z1 and $\mathrm{J} 1$ are ranked among the likely low- $z$ contaminants based on the detections in the $24 \mu \mathrm{m}$ and $z_{850}$ bands, respectively.

\subsection{Physical properties from SED fits}

From our SED fits using the Bruzual \& Charlot templates, we can also derive the physical properties of the galaxies, such as the age of the stellar population, the stellar mass, star-formation rate, and attenuation. The resulting masses, SFR, and attenuation $A_{\mathrm{V}}$ (derived assuming the Calzetti law) and the uncertainties, derived from 1000 Monte Carlo simulations of each object, are shown in Figs. 8 and 9. For comparison, we also show the properties of $z \sim 6-8$ galaxies analyzed by SB2010 using the same SED fitting tool.

As can be seen, the masses derived for the most likely high$z$ candidates based on photometric redshifts with a luminosity prior, namely Y3, Y4, and Y5, are among the highest masses found by SB2010, typically of the order of $M_{\star} \sim 2 \times 10^{10}$ to $2 \times$ $10^{11} M_{\odot}$. Including relatively large corrections for attenuation

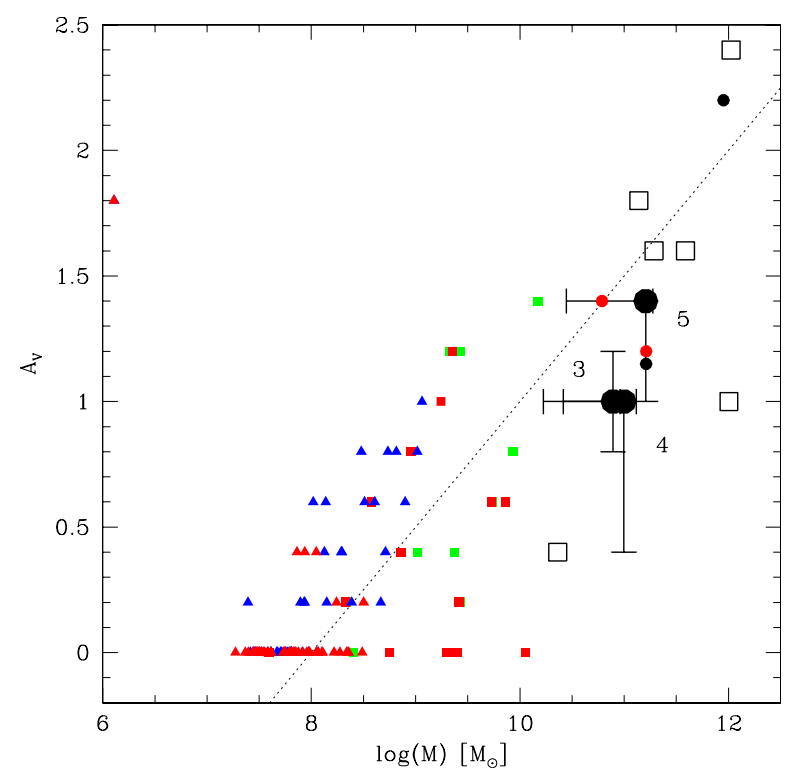

Fig. 9. Mass $-A_{\mathrm{V}}$ relation for the same objects as shown in Fig. 8 (same symbols).

$\left(A_{\mathrm{V}} \sim 0.4-1.4\right)$, their SFR range from $\sim 100$ to $\sim 10^{3} M_{\odot} \mathrm{yr}^{-1}$. For comparison, the SFR derived from the rest-frame UV luminosity $L_{1500}$ using the Kennicutt (1998) calibration is typically $\sim 100 M_{\odot} \mathrm{yr}^{-1}$ for these sources (see Table 4).

When including the full sample of optical-dropout candidates, the masses and SFRs achieved are much larger, reaching $M_{\star} \sim 10^{12} M_{\odot}$ in most cases, and even $M_{\star}>10^{12} M_{\odot}$ for the brightest $Y$-drop candidate, Y1, if at high-z. However, for the most extreme objects the uncertainties in $A_{\mathrm{V}}$ and SFR are very large. For objects with well-defined errors, the SFR may reach up to $2000-3000 M_{\odot} \mathrm{yr}^{-1}$. Interestingly enough, these values are not inconsistent with the trends found by SB2010 for the fainter $z>6$ galaxies from recent HST surveys, if extrapolated to higher masses. These properties are also similar to those of the two $z \sim 7$ galaxy candidates found by Capak et al. (2011) in the COSMOS wide field survey. However, whether these relatively bright objects are truely high redshift galaxies, hence objects with such extreme properties, remains of course questionable (see below).

Two of our optical dropout galaxies ( $\mathrm{z} 1$ and $\mathrm{Y} 7$ ) are detected by MIPS at $24 \mu \mathrm{m}$ with fluxes of 3.4 and $1.1 \times 10^{-4} \mathrm{Jy}$ respectively, and we have non-detection constraints for three additional sources included in the MIPS image (Y3, Y4, and J1, with $1 \sigma$ fluxes below $38.7 \mu \mathrm{Jy}$ ), whereas $\mathrm{Y} 8$ is highly contaminated by neighbouring galaxies (see Fig. 11). Two objects of this sample have also been detected with Herschel and LABOCA between 160 and $870 \mu \mathrm{m}$ (Boone et al., in prep.). This data identifies z1 and Y5 as mid- $z$ interlopers.

For $z \sim 1-2$ galaxies, the MIPS band probes a region in the mid-IR corresponding or close to redshifted PAH emission. If at high redshift $(z \sim 7-9)$, the $24 \mu \mathrm{m}$ band samples the region between $2-3 \mu \mathrm{m}$. To illustrate the overall SED of our MIPS-detected (or constrained) objects, we show their photometry together with several SED fits in Fig. 10. In addition to our standard spectral templates with/without nebular emission, we also show best-fits using (semi)empirical templates of nearby galaxies including in particular very dusty galaxies, sucha as LIRGs, and ULIRGs. In practice, we used the templates from the GRASIL models of Silva et al. (1998), the SWIRE starburstAGN templates of Polletta et al. (2008), and the LIRG-ULIRG 
$\mathrm{z} 1$
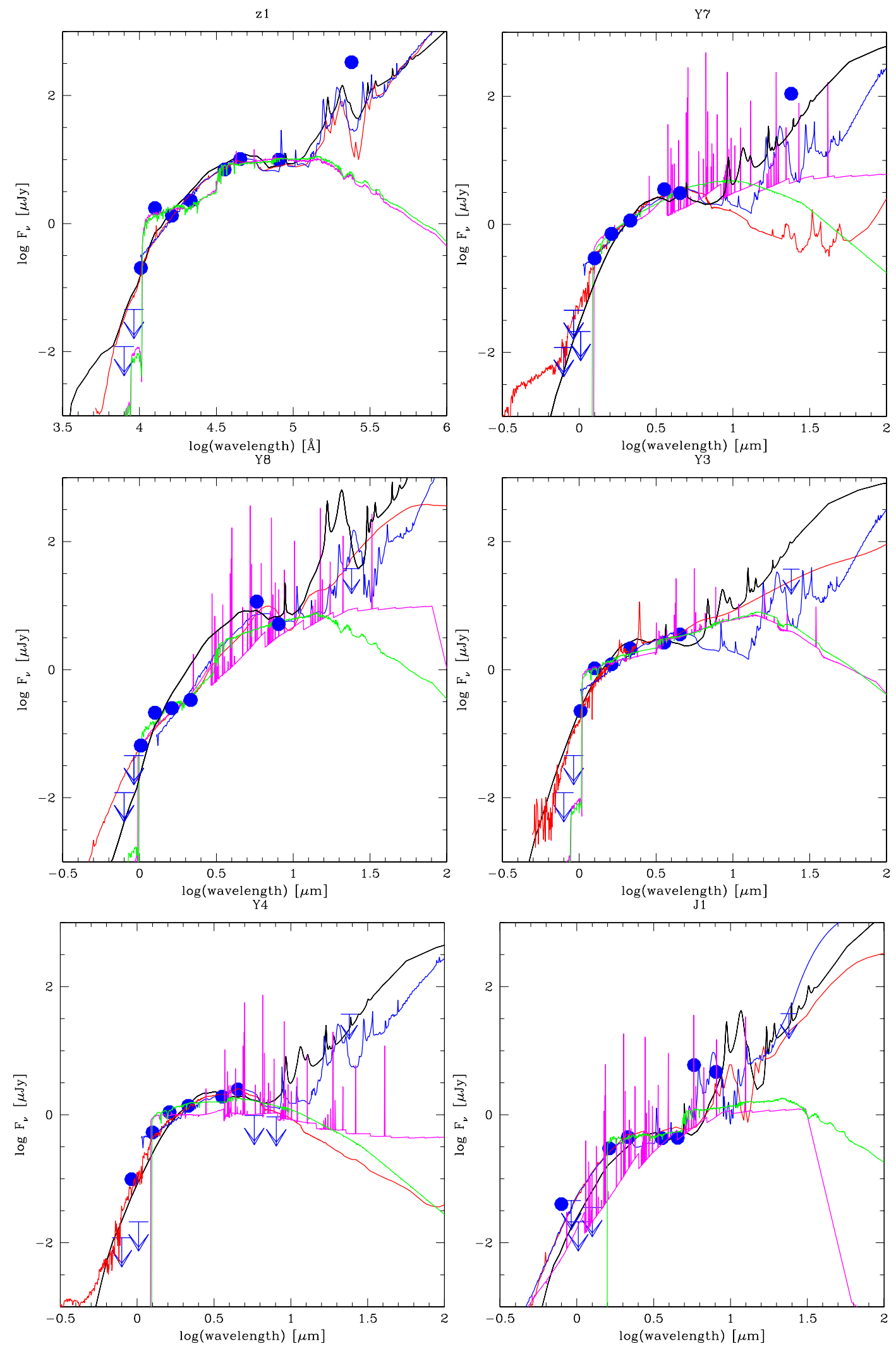

Fig. 10. Best-fit SEDs for candidates with available constraints at $24 \mu \mathrm{m}$ : z1 and Y7 (sources clearly detected, top row), Y8 (source highly contaminated, middle left panel), and Y3, Y4, and J1 (non-detection, $1 \sigma$ flux $<38.7 \mu \mathrm{Jy}$ ). Different spectral templates are displayed. Green (pink) lines show the best-fit solutions at $z>7$ using our standard templates based on Bruzual \& Charlot models without (with) nebular lines. Black and red lines show the best-fits at low redshift $(z<4)$ using the templates from the GRASIL models (Silva et al. 1998) and the SWIRE starburst-AGN templates of Polletta et al. (2008), respectively. Blue lines show best-fit solutions using the empirical LIRG-ULIRG templates of Rieke et al. (2009). 


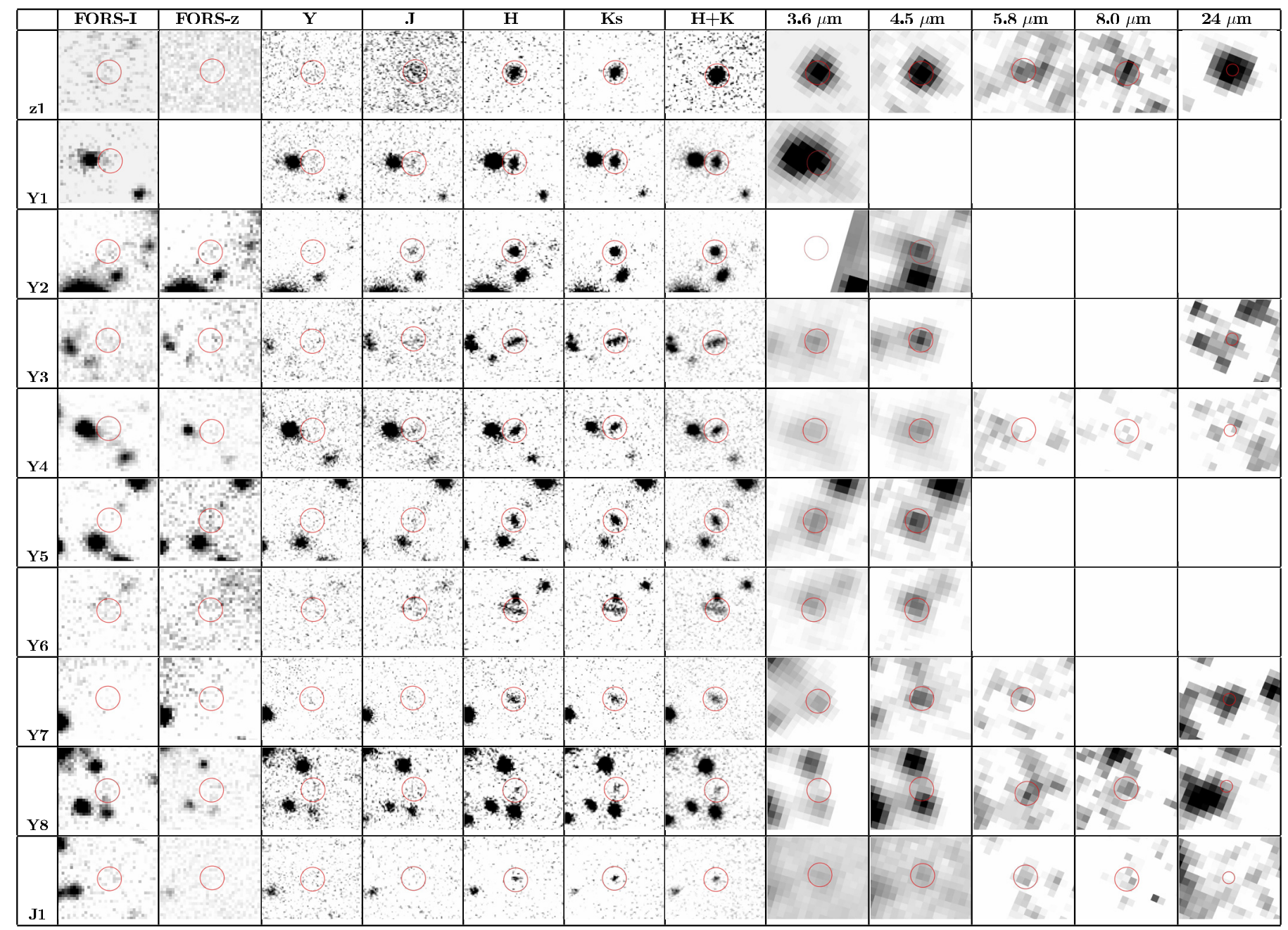

Fig. 11. Thumbnail images of the bright $z, Y$, and $J$-dropouts found in A2667, covering a $9^{\prime \prime} \times 7.3^{\prime \prime}$ area of the original images from $I$ band to $8.0 \mu \mathrm{m}$, and $18^{\prime \prime} \times 14.6^{\prime \prime}$ for MIPS $24 \mu \mathrm{m}$. The position of each candidate is displayed by a circle of $2^{\prime \prime}$ diameter aperture. Images are displayed in linear scale ranging between -5 and $20 \sigma$ around the sky background.

templates of Rieke et al. (2009); redshift and additional extinction were kept as free parameters in the fit. For all of these models, we here show the best-fits under the constraint that $z<4$ but without any additional constraint. We note that the best-fit photometric redshifts at low- $z$ can differ quite significantly depending on the set of spectral templates used and between the objects. For example, we find that $z_{\text {phot }} \sim 0.1,1.5$, and 2.7 for $\mathrm{Y} 3$; $1.6-1.7$ for $\mathrm{Y} 7,0.02,1.6$, and 1.6 for $\mathrm{Y} 8$, and $0.1-0.5$ for $\mathrm{J} 1$. Furthermore, the $24 \mu \mathrm{m}$ flux expected from these fits can vary by more than an order of magnitude. Interestingly fits at high$z$ show fluxes at $24 \mu \mathrm{m}$ that are comparable to those expected from some of low- $z$ best-fits, thus providing weak additional constraints on the redshift.

Several objects in this sample deserve additional specific comments:

- z1: this is the brightest source in our sample. It is an extended isolated object, clearly non-stellar. It is detected in all filter bands between $Y$ and $24 \mu \mathrm{m}$ except at $5.8 \mu \mathrm{m}$ where no data is available. Although the best-fit is obtained at high-z, even with a luminosity prior, the $24 \mu \mathrm{m}$ flux seems incompatible with the high- $z$ solution (see Fig. 10). It even appears too bright in this band compared to the best-fit templates at low- $z$. However, the observed flux in the $J$-band, and that, if at low- $z$, it should also be detected in the $I$ and $z$ bands at $\geq 3 \sigma$ level (given the depth of our survey), remains difficult to explain;

- Y3: although the $24 \mu \mathrm{m}$ non-detection of this object helps us to exclude at least one of the GRASIL templates at low- $z$, it is still consistent with other templates at low and high-z. The main failure of the low- $z$ templates is probably again the mismatch between $J$ and the optical bands;

- Y4: as for Y3, the upper limit detection at $24 \mu \mathrm{m}$ provides weak additional constraints on the redshift. The nondetections at 5.8 and $8 \mu \mathrm{m}$ are interesting because both solutions at low and high- $z$ seem to predict a detection at the $\sim 2-3 \sigma$ level. The object is formally detected at the $\lesssim 2 \sigma$ level in the $z_{1}$ band, but not detected in the $z_{2}$ image;

- Y7: for this object, the expected $24 \mu$ m fluxes for the best-fit at high and low redshifts are very similar. In particular, the flux of the $z \sim 9$ fit is even quite similar to the brightest flux predicted for dusty galaxy templates at $z \sim 1.6$. Therefore, this object cannot be excluded as a genuine high- $z$ based on its MIPS photometry without introducing a luminosity prior. 
In addition, the observed $24 \mu \mathrm{m}$ flux seems too high irrespective of the template redshift for a relatively isolated source (see Fig. 11);

- Y8: the 5.8 and $8 \mu \mathrm{m}$ photometry for this object could be seriously contaminated by bright neighboring sources (see Fig. 11), although this seems to be the only emission at this precise location. The $24 \mu \mathrm{m}$ flux shown in Fig. 10 corresponds to the lower limit, and is compatible with both the low and the high- $z$ solutions;

- J1: this is the faintest candidate in our sample, and the only one that is located in the central area covered by the HST. The best-fit solution is found at high- $z$ even when including the $z_{850}$-band flux and a luminosity prior, as seen in Table 4. However, the detection in the $z_{850}$-band makes the high- $z$ solution unlikely. The upper limit detection at $24 \mu \mathrm{m}$ provides weak additional constraints on the redshift. The detection on the HST image is fully consistent with the large break identified in ground-based images. The apparent strong "doublebreak" between the optical and near-IR and between 4.5 and $5.8 \mu \mathrm{m}$ seems quite unusual. High- $z$ solutions have difficulty reproducing the latter break (see Fig. 6), while low- $z$ solutions predict a flux excess in the optical domain. The break at $4.5-5.8 \mu \mathrm{m}$ could be explained by PAH features boosting the 5.8 and $8 \mu \mathrm{m}$ fluxes, as shown in Fig. 6 . This is one of the candidates for which a spectroscopic follow-up is needed to be able to draw firm conclusions.

\subsection{Contamination level based on stacked images}

On the basis of previous discussions, a large fraction of opticaldropout galaxies in this sample could be low- $z$ contaminants, reaching as high as $\sim 70 \%$ based on luminosity priors. To quantify this estimate more accurately, we generated stacked images in the $I$ and $z$ bands, where genuine high- $z$ galaxies are not expected to be detected. In contrast, the $S / N$ achieved on the stacked image should allow us to estimate the contamination level.

For each candidate, a $10^{\prime \prime} \times 10^{\prime \prime}$ region has been selected in the $I$ and $z$-band images around the centroid position on the detection image $H+K \mathrm{~s}$. An additive zero level correction has been applied to each single image to properly remove the local average sky background. I and $z$-band images have been averaged using IRAF routines and different pixel rejection schemes in order to improve the suppression of neighboring sources on the final combined images. Due to the presence of a closeby galaxy, we do not include the Y1 field in the final stacks. We have checked that the sky backgound noise on the final combined images actually improves as expected as a function of the total number of images stacked, i.e. reaching a reduction by a factor close to 3 on the final stacks with respect to the original images.

We used IRAF routines to measure fluxes and magnitudes in a $1.3^{\prime \prime}$ diameter aperture, together with the corresponding error bars in the different stacks. The highest $S / N$ is achieved in the $I$-band, where we measure up to $I=28.0 \pm 0.3$ for the combined source, i.e. a $S / N \sim 3$. The detection in the $z$-band is less significant, reaching $z=27.7 \pm 0.5(S / N \sim 2)$ in the best-detected final stack. These results confirm that there is indeed some contamination by low $-z$ interlopers in our sample. The detection level in the $I$-band is roughly consistent with $\sim 70 \%$ of the sample being detected at the $\sim 1 \sigma$ level, although 3-4 objects detected between 1 and $2 \sigma$ would be enough to account for the signal in this band. The flux measured in the $z$-band seems to favour either a signal below $1 \sigma$ for a large majority of our candidates, or a higher signal coming from a small fraction of contaminants (between $\sim 20 \%$ with $S / N \lesssim 2$, or $\sim 30 \%$ with a mean $S / N \sim 1.5$ ).

\section{Discussion}

As the selection diagrams (Figs. 2 and 3) show, most of our bright candidates have near-IR colors distinguishing them clearly from normal galaxies at low redshift and late-type stars. In addition SED-fitting results clearly favor a high- $z$ solution for all these candidates, irrespective of their intrinsic luminosities. However, as seen in Table 4, high- $z$ solutions yield magnification-corrected luminosities, which typically range between 3 times (Y8) and 40 times (z1, Y2) $L_{1500}^{*}$ at these redshifts, according to the evolving LF by Bouwens et al. (2008), suggesting a potential contamination by low- $z$ interlopers. In this section, we discuss the observed versus expected number counts of high- $z$ galaxies, and the possible sources of contamination in our survey. We also compare our results and the properties of this sample with those obtained by previous authors using similar techniques.

\subsection{Observed versus expected number density of high-z sources}

We first compute the expected number counts of bright high- $z$ sources in this lensing field, as a function of redshift and for the range of magnitudes of our candidates (i.e. $A B \sim 23$ to 25.5), and we compare these numbers with current observations. This calculation was done following the same procedure as in Maizy et al. (2010). All the noisy regions in the field, in particular around bright galaxies in the cluster core, have been masked. The presence of a strong lensing cluster in this field introduces two opposite effects on the number counts as compared to blank fields. Gravitational magnification increases the number of faint sources by improving the detection towards the faint end of the $\mathrm{LF}$, whereas the dilution effect reduces the effective volume by the same factor. As discussed in Maizy et al. (2010), the difference between lensing and blank field results depends strongly on the shape of the LF. We expect lensing clusters to be more efficient than blank fields in relatively shallow surveys.

Number counts of sources brighter than a limiting magnitude were computed by a pixel-to-pixel integration of the magnified source plane as a function of redshift (see Eq. (6) in Maizy et al. 2010), for redshift bins $\Delta z=1$, using the evolving LF by Bouwens et al. (2008), i.e. with the Schechter parameters directly derived from Eq. (3) in their paper. For comparison purposes, we also derive the expected counts with the Beckwith et al. (2006) LF for $z \sim 6$ galaxies, assuming no evolution. This LF displays the same Schechter parameters as for Steidel et al. (2003) and Reddy \& Steidel (2009), but the normalization factor is three times smaller than for Steidel et al. (2003). Table 5 summarizes these results for two different limiting magnitudes, $A B<25.5$, where all the current candidates are found, and $A B<26.0$, which corresponds to our $\sim 5 \sigma$ detection level (in the filter spanning the UV region around $1500 \AA$ ). Error bars include Poisson uncertainty and field-to-field variance, following Trenti \& Stiavelli (2008). Given the small number counts expected, these fluctuations dominate the error budget (see also the discussion in Maizy et al. 2010). We also computed number counts using the latest Schechter parameters presented by Bouwens et al. (2010) for galaxies at $z \sim 7$ in the $z=[7.0,8.0]$ interval, and for galaxies at $z \sim 8$ in the $z=[8.0,9.0]$ interval (identified by $*$ in Table 5). The changes with respect to the 2008 version are relatively minor in this case given the luminosity domain, which is largely dominated by statistical uncertainties.

Candidates were selected on a total survey (clean) area of $\sim 42 \operatorname{arcmin}^{2}$ ( $33 \operatorname{arcmin}^{2}$ when corrected for dilution), i.e. an 
Table 5. Expected number counts with different LF assumption

\begin{tabular}{lcccc}
\hline \hline & LF(1) & & LF(2) & \\
Redshift & Blank & A2667 & Blank & A2667 \\
\hline$A B<25.5$ & & & & \\
{$[6.0,7.0]$} & $0.5(1)$ & $1.5(2)$ & $2.9(3)$ & $4.2(3)$ \\
{$[7.0,8.0]$} & $<0.1(1)$ & $0.3(1)$ & $1.5(2)$ & $2.3(2)$ \\
$(*)$ & $0.1(1)$ & $0.5(1)$ & & \\
{$[8.0,9.0]$} & $<0.1(1)$ & $0.1(1)$ & $0.8(1)$ & $1.4(2)$ \\
$(*)$ & $<0.1(1)$ & $0.2(1)$ & & \\
{$[9.0,11.0]$} & $<0.1(1)$ & $0.1(1)$ & $0.6(1)$ & $1.3(2)$ \\
\hline$A B<26.0$ & & & & \\
{$[6.0,7.0]$} & $3.1(3)$ & $5.4(4)$ & $8.5(5)$ & $10.5(5)$ \\
{$[7.0,8.0]$} & $0.4(1)$ & $1.1(2)$ & $4.9(3)$ & $6.4(4)$ \\
$(*)$ & $0.8(1)$ & $1.9(2)$ & & \\
{$[8.0,9.0]$} & $<0.1(1)$ & $0.3(1)$ & $2.9(3)$ & $4.0(3)$ \\
$(*)$ & $0.3(1)$ & $0.7(1)$ & & \\
{$[9.0,11.0]$} & $<0.1(1)$ & $0.1(1)$ & $2.8(2)$ & $4.3(3)$ \\
\hline
\end{tabular}

Notes. Number counts are computed for sources with $A B<25.5$ and $A B<26.0$ expected in the field of A2667, with and without the presence of a lensing cluster, for different redshift bins and for two different LFs: Bouwens et al. (2008) (1) and Beckwith et al. (2006) (2) (see text). Error bars in number counts, including Poisson uncertainty and field-tofield variance, are given in brackets.

effective lensing-corrected comoving volume per unit $\Delta z=1$ ranging between 7.4 and $4.9 \times 10^{4} \mathrm{Mpc}^{-3}$ (covolume). For comparison with the current sample, we consider four redshift bins, the same presented in Table 5, and we bin the candidates as follows (cf. Table 4): z1, Y1, Y3, Y6 and Y8 (i.e. five objects) in the bin [7.0, 8.0], Y2, Y5 (two objects) in the bin [8.0,9.0], and Y4, Y7 (two objects) in the bin $[9.0,11.0]$. $\mathrm{J} 1$ is also in this last bin, but the high- $z$ hypothesis is in this case highly unlikely.

Assuming the evolving LF by Bouwens et al. (2008) or Bouwens et al. (2010), we expect up to a maximum of one or two sources at $z \sim 7.0-8.0$ in this wide field with $A B<25.5$, and typically between two and ten sources with the Reddy \& Steidel (2009) LF, all of them within $24.5<A B<25.5$. In our sample, only two out of the five $z \sim 7.5$ candidates are included in this magnitude interval (Y6 and Y8), in full agreement with expectations for an evolving LF, whereas z1, Y1, and Y3 seem too bright. On the other hand, only $\mathrm{Y} 3$ qualifies as a high- $z$ candidate when using a luminosity prior (see Sect. 4.5). This result is also consistent with the expectations for a Beckwith et al. (2006) LF.

Regarding the number of bright $(24.5<A B<25.5)$ higherredshift sources expected at $z \approx 8.5$ and $z \approx 10$ in this field, it ranges between a maximum of one per bin for an evolving LF, and typically between one and seven 7 per bin with a constant $z \sim 3 \mathrm{LF}$, when including the error bars. Only Y7 and J1 seem to be in agreement with the predictions of both the redshift interval and observed magnitude, whereas Y2, Y4, and Y5 seem too bright when taken at face values to be all at such high redshifts, i.e. we have less than a one in six chance of finding one such an intrinsically bright object in this field, even when assuming no evolution in the LF since $z \sim 4$. On the other hand, only Y4 and Y5 qualify as high- $z$ candidates when using a luminosity prior to derive photometric redshifts (see Sect. 4.5), i.e. a maximum of two candidates per bin. The result is the same when blindly excluding the brightest candidates, as well as J1 for arguments related to its SED (cf. above). Our results seem to be in agreement with an evolving LF, and also be consistent with Beckwith et al. (2006) counts at $z \sim 6$ when error bars in number counts are taken into account.

In summary, our sample includes some intrinsically bright sources (six out of ten) for which the best-fit photometric redshifts seem difficult to reconcile with the LF previously measured at high- $z$, even when no evolution is assumed. When these sources are blindly excluded, however, or when the sample is restricted to galaxies surviving a stringent prior in luminosity (namely Y3, Y4, and Y5), observed number counts at $z \gtrsim 7.5$ are in agreement with expectations for an evolving LF, which is also consistent within the error bars with a constant LF since $z \sim 6$, and inconsistent with a constant LF since $z \sim 4$.

\subsection{Contamination}

As seen in Sect. 5.1 based on pure LF and counts arguments, six out of ten candidates, seem too bright (or their abundance is too high) relative to expectations. In addition half of our sample could be identified as mid- $z$ interlopers when computing photometric redshifts including a luminosity prior, and two galaxies among the surviving sample can be excluded based on their SED properties ( $\mathrm{z} 1$ and $\mathrm{J} 1$ ), leading to a contamination level close to $70 \%$. We discuss below the possible sources of contamination, which must also be present in other current Lyman break surveys.

The selection windows of several objects are relatively close to the boundaries. Object Y8, which also has a high stellarity index, has colors indistinguishable within the errors from latetype stars (cf. also Fig. 4). However, although its flux could not be properly extracted due to blending, it seems to be detected at $24 \mu \mathrm{m}$, which excludes it being a Galactic late-type star. It is therefore, most likely to be a dusty, low-redshift galaxy (cf. Fig. 10). Objects Y3 and Y6 are also relatively close to the boundaries of the Y-drop selection box, where stars and lowredshift galaxies lie. However, when colors spanning a wider wavelength range are considered, as in Fig. 4, the difference from "contaminants" is more pronounced, especially for Y3.

As Fig. 4 shows, four out of nine sources (z1, Y2, Y3, Y5) with $z$-band photometry have colors $(z-J) \geq 3$ that are redder than the most extreme low-redshift objects compiled by Capak et al. from the large field COSMOS survey. Six of our ten objects (z1 and Y1-Y5) also show $(I-J)>4$, i.e. the depth needed in the z-band to robustly select $z>7$ galaxies, according to the comparison with COSMOS galaxies (cf. Capak et al. 2011). In addition, our data also included the $Y$-band, yielding a stronger constraint on the shape of the SED than available e.g. for the COSMOS sample.

In other color-color diagrams, such as $(J-K)$ versus $(J-3.6)$ examined by Stanway et al. (2008) and Capak et al. (2011), our objects are where expected for high- $z$ objects, and they show similar colors as the two $z>7$ candidates of Capak et al. (2011). The (3.6-4.5) color is also as expected from these papers.

Could emission-line objects similar to the ultra-strong emission-line galaxies (USELs) discovered by Kakazu et al. (2007) at $z \sim 0.3-1.5$ contaminate our sample? In principle, their strong $\mathrm{O}[\mathrm{III}] \lambda \lambda 4959,5007, \mathrm{H} \alpha$, and other emission lines could lead to blue $(J-H)$ colors and a spectral break if indeed they reside at $z \sim 1.6-1.8$, as expected for high- $z$ galaxies. However, from the object properties of the USELs known so far, for several reasons this seems quite unlikely. If we assume a rest-frame equivalent width of $\sim 1000 \AA$ for both O [III] $\lambda 5007$, and $\mathrm{H} \alpha$, as observed for the most extreme objects (cf. Kakazu et al. 2007), their contribution to the broad band $J$ and $H$ filters (with widths 
of $\Delta \lambda \sim 1400-2700 \AA$ ) should be at best $\sim 35-50 \%$. Assuming a flat underlying continuum (in $F_{v}$ ), as expected for strongly star-forming objects with little/no extinction and roughly also consistent with their observed colors (cf. Kakazu et al. 2007; $\mathrm{Hu}$ et al. 2009), these emission lines can therefore not mimick a spectral break much larger than $\sim 0.75 \mathrm{mag}$. Hence, these objects, if they exist at $z \sim 1.6-1.8$, are most likely unable to reproduce the break of $(Y-J) \gg 1$ shown by most of our objects.

Our spectral models allowing also for the presence of nebular emission (lines and continua) find indeed some extreme best-fit templates when $z<4$ is imposed. This is for example the case for $\mathrm{Y} 8$ and $\mathrm{J} 1$, where our fitting procedure exploring a wide range of parameter space identifies relatively young $(<10 \mathrm{Myr})$ objects with a very strong extinction $\left(A_{\mathrm{V}} \sim 3-3.8\right)$ as the best-fits at low redshift, as shown in Fig. 6. These very unusual and probably unrealistic examples illustrate the difficulty in reproducing the strong spectral break present in our objects using strong emission line galaxy spectra at low redshift. In any case, were the near-IR photometry of our relatively bright objects to be strongly contaminated by emission lines, these should be detectable with current instruments.

Two of our optical dropout galaxies (z1 and Y7) are detected by MIPS at $24 \mu \mathrm{m}$, leading to a preferential identification as mid$z$ interlopers, and we have constraints for four additional sources (Y3, Y4, Y8, and J1, see Sect. 4.6). However, as discussed in Sect. 4.6, $24 \mu \mathrm{m}$ fluxes cannot help us to distinguish between high and low- $z$ solutions for a majority of our candidates.

To understand the nature of these possible contaminants, we compared the present candidates/counts with the results found for our blank-field survey WUDS (WIRCAM Ultra Deep Survey $^{5}$; Pelló et al., in prep.). WUDS is an extremely deep photometric survey with WIRCAM at CFHT over $400 \mathrm{arcmin}^{-2}$ on the CFHTLS Deep pointing D3, using the same four filter-bands $Y J H K$ s as in this survey, and robust non-detection constraints in the optical bands (i.e. $\operatorname{ugriz}(A B) \sim 27$ to 28.3 at $3 \sigma$ level, depending on filters). The main advantage of WUDS with respect to the present survey is the large field of view and the wavelength coverage shortwards of the $i$-band. The depth in the nearIR bands is lower, reaching $Y J \sim 25.8$ and $H K \mathrm{~s} \sim 25.3(3 \sigma)$. When applying the same selection function introduced here for the $Y$-dropouts (in both optical and near-IR bands), 13 candidates are retained over the WUDS field after visual inspection, and among them 7 candidates in the $H_{A B} \sim 23.0-24.0$ interval, which is identical to that from which our five "bright" candidates are found. When we apply a more restrictive non-detection criterium in the optical bands based on the full ugriz domain (detection below $2 \sigma$ in all filters), only three candidates survive, all of them are within the $H_{A B} \sim 23.0-24.0$ interval. Two of these WUDS candidates display the same properties as the $z \geq 7.5 \mathrm{ob}-$ jects in the HAWK-I field in terms of photometric redshifts and $P(z)$ distributions, the third one being more dubious (it has a degenerate solution between low $-z$ and high $-z$ ). This means that a more robust non-detection in the optical bands bluewards of to the $I$-band could have removed between $\sim 50 \%$ and $75 \%$ of our present candidates in the HAWK-I field.

In summary, the contamination in this field comes essentially from mid- $z$ interlopers, with a negligible contribution from latetype stars. In addition strong emission-lines seem unable to reproduce the large breaks observed. Only a young stellar population together with a strong extinction provide a reasonable fit at $z<4$. Based on the comparison with the blank-field survey
WUDS, and assuming that the nature of contaminant sources is the same in all fields, we might have removed between $\sim 50 \%$ and $75 \%$ of the present sample with a wider wavelength coverage in the optical bands bluewards of the $I$ band.

\subsection{Comparison with previous results}

We compare the number densities and properties of $z \geq 7.5$ candidates in this sample with those obtained by previous authors using similar techniques to explore this redshift domain. A direct comparison is difficult given the different selection functions.

Our selection criterium (c) is the same adopted by Capak et al. (2011) except for the $K \mathrm{~s}-4.5 \mu \mathrm{m}>0$ condition (due to partial coverage of the HAWK-I field of view), making the comparison easier in this case. All our $Y$-dropout candidates fulfil their color selection, except for Y4, which is formally detected in the $z_{1}$ band at the $\$ 2 \sigma$ level. However, all of them are too faint to be included in their sample (i.e. $J<23.7$, their $5 \sigma$ detection level), except for $\mathrm{z} 1$. This object, once corrected for magnification, is also $\sim 0.3$ to $0.5 \mathrm{mag}$ fainter than all their retained candidates (depending on the candidate and filter). In other words, the density of bright high- $z$ candidates in our field is consistent with the density derived by Capak et al. (2011), leading to a weak constraint on the density $<3 \times 10^{-5} \mathrm{Mpc}^{-3}$ for $M_{1500} \sim-23 \mathrm{ob}-$ jects.

The colors and SEDs of present candidates are consistent with the selection functions introduced by Bouwens et al. (2008, 2010) in the GOODS, HUDF, HDF South, and lensing fields. All our candidates fulfil their $z \geq 7$ preselection when using equivalent filter-bands, i.e. our ground-based filters instead of $z_{850}$ and $H_{160}$ filters. We note however that even $\mathrm{J} 1$, which is detected in the $z_{850}$ filter, remains in the sample because of its large break $\left(z_{850}-H=2.2\right)$. All our candidates except $\mathrm{J} 1$ (which is not detected in the $J$-band) fulfil their $z \sim 7 z J H$ selection function, as well as the selection introduced by Hickey et al. (2009) for $6 \lesssim z \lesssim 9$ galaxies. Instead, J1 satisfies the $z \sim 9 J H$ selection function by Bouwens et al. (2008, 2010; see also Fig. 3), although the detection in the $z_{850}$ band excludes it as a genuine $z \sim 9$ candidate. In addition, two of the five candidates detected in the $Y$-band (z1 and Y3) fulfil the rough selection defined by Ouchi et al. (2009) for $z \sim 7$ candidates, but they are not included when applying the selections proposed by Wilkins et al. (2010) or Castellano et al. (2010), namely $z-Y>1.2(1.0)$ and $Y-J<2.0$. Our candidates are indeed slightly redder in $Y-J$, which is consistent with all our sources having photometric redshifts $z \gtrsim 7.5$. In summary, all the present candidates would have been selected by the usual functions targeting $z \geq 7.5$ sources based on broad-band colors.

The magnitudes of only two of our candidates, namely Y8 and $\mathrm{J} 1$, are found in the range covered by Bouwens al. (2010) in their survey of the GOODS field, i.e. $H_{A B} \sim 25.5-26.0$ once corrected for magnification. At this depth level, our number counts of $z \geq 7.5$ candidates are $\sim 0.06$ sources $\operatorname{arcmin}^{-2}$, in good agreement with their previous findings within the same magnitude interval.

The main difference with respect to previous studies is the presence of several "bright" $M_{1500} \sim-23.0$ candidates at $z \geq$ 7.5 that cannot be easily excluded based on broad-band colors and photometric redshifts (see also Sect. 5.2 above), unless a luminosity prior is used in addition.

${ }_{5}$ http://regaldis.ast.obs-mip.fr/ 


\section{Conclusions}

The photometric survey conducted on A2667 has allowed us to identify $10 z, Y$, and $J$-dropout galaxies in the selection windows targeting $z \geq 7.5$ candidates within the $\sim 7^{\prime} \times 7^{\prime}$ HAWK-I field of view ( $33 \mathrm{arcmin}^{2}$ of effective overlapping area in all selection bands). All of them are detected in the $H$ and $K$ s bands, in addition to the $J$ and/or IRAC $3.6 \mu \mathrm{m} / 4.5 \mu \mathrm{m}$ images, with $H_{A B}$ ranging from 23.4 to 25.2 , and modest magnification factors between 1.1 and 1.4. SED-fitting results in all cases yield a best solution at high- $z$ ( $z \sim 7.5$ to 9$)$, with a less significant solution at low $-z(z \sim 1.7$ to 2.8$)$. However, several of these sources seem too bright to be at $z \geq 7.5$, suggesting strong contamination by low- $z$ interlopers which must also be present in other current Lyman break surveys.

A broad and deep wavelength coverage in the optical bands allows us to suppress the majority of low- $z$ interlopers. Indeed, based on a comparison with the WUDS survey, we have estimated that a fraction of $\sim 50-75 \%$ of our bright candidates could be (extreme) low- $z$ interlopers. The same result is achieved when photometric redshifts are computed using a luminosity prior. In this case, only half of the sample survives, and only three objects (namely Y3, Y4, and Y5) are finally retained when including all the available information about the SED presented in this paper. These low- $z$ interlopers, which cannot be easily identified based on broad-band photometry in the optical and near-IR domains alone, are indeed rare objects, in the sense that they are not well described by current spectral templates given the large break. A reasonable good fit for these objects at $z<4$ is obtained assuming a young stellar population together with a strong extinction. On the other hand, at least 1 and up to 3 sources in our sample are expected to be genuine high- $z$ objects. Spectroscopy is needed to ascertain their redshift and nature. Some of them could also be detected in the IR or sub-mm bands given the estimated dust extinction and high SFRs. Indeed, two sources in this sample, z1 and Y5, have been detected in the Herschel PACS \& SPIRE bands and LABOCA, making the high- $z$ identification highly unlikely (Boone et al., in prep.).

Only one source (A2667-z1) fulfils the color and magnitude selection criteria of Capak et al. (2011), although it is $\sim 0.4 \mathrm{mag}$ fainter than their candidates once corrected for magnification. Its $24 \mu \mathrm{m}$ flux seems incompatible with a high- $z$ identification, although the observed flux in the $J$-band, and the non-detection in the $I$ and $z$ bands seem difficult to reconcile with a low- $z$ galaxy.

After removing the brightest candidates, based on luminosity priors and SED properties, the observed number counts of $z \gtrsim$ 7.5 candidates in this field seem to be in good agreement with expectations for an evolving LF, and also consistent within the error bars with a constant LF since $z \sim 6$. In contrast, they are inconsistent with a constant LF since $z \sim 4$.

Acknowledgements. Part of this work was supported by the French Centre National de la Recherche Scientifique, the French Programme National de Cosmologie et Galaxies (PNCG), as well as by the Swiss National Science Foundation. We acknowledge support for the International Team 181 from the International Space Science Institute in Berne. J.R. acknowledges support from a EU Marie-Curie fellowship. This work recieved support from Agence Nationale de la recherche bearing the reference ANR-09-BLAN-0234. This paper is based on observations collected at the European Space Observatory, Chile (71.A-0428, 082.A-0163).

\section{References}

Beckwith, S. V. W., Stiavelli, M., Koekemoer, A. M., et al. 2006, AJ, 132, 1729 Benitez, N. 2000, ApJ, 536, 571

Bertin, E., \& Arnouts, S. 1996, A\&AS, 117, 393

Bolzonella, M., Miralles, J. M., \& Pelló, R. 2000, A\&A, 363, 476

Bouwens, R. J., Thompson, R. I., Illingworth, G. D., et al. 2004, ApJ, 616, 79

Bouwens, R. J., Illingworth, G. D., Blakeslee, J. P., \& Franx, M. 2006, ApJ, 653, 53

Bouwens, R. J., Illingworth, G. D., Franx, M., \& Ford, H. 2008, ApJ, 686, 230 Bouwens, R. J., Illingworth, G. D., Bradley, L. D., et al. 2009, ApJ, 690, 1764 Bouwens, R. J., Illingworth, G. D., Oesch, P. A., et al. 2010a, ApJ, 709, L133

Bouwens, R. J., Illingworth, G. D., Oesch, P. A., et al. 2010b, ApJ, submitted [arXiv: 1006.4360 ]

Bradley, L. D., Bouwens, R. J., Ford, H. C., et al. 2008, ApJ, 678, 647

Bruzual, G., \& Charlot, S. 1993, ApJ, 405, 538

Bruzual, G., \& Charlot, S. 2003, MNRAS, 344, 100

Bunker, A. J., Geballe, T. R., Leggett, S. K., Kirkpatrick, J. D., \& Golimowski, D. A. 2004, MNRAS, 355, 374

Burgasser, A. J., Burrows, A., \& Kirkpatrick, J. D. 2006, ApJ, 639, 1095

Calzetti, D., Armus, L., Bohlin, R. C., et al. 2000, ApJ, 533, 682

Capak, P., Mobasher, B., Scoville, N. Z., et al. 2011, ApJ, 730, 68

Castellano, M., Fontana, A., Boutsia, K., et al. 2010, A\&A, 511, A20

Coleman, D. G., Wu, C. C., \& Weedman, D. W. 1980, ApJS, 43, 393

Covone, G., Kneib, J.-P., Soucail, G., et al. 2006, A\&A, 456, 409

Cuby, J. G., Le Fèvre, O., McCracken, H., et al. 2003, A\&A, 405, L19

Cuby, J. G., Hibon, P., Lidman, C., et al. 2007, A\&A, 461, 911

Daddi, E., Dickinson, M., Morrison, G., et al. 2007, ApJ, 670, 156

Egami, E., Kneib, J.-P., Rieke, G. H., et al. 2005, ApJ, 618, L5

Egami, E., Misselt, K. A., Rieke, G. H., et al. 2006, ApJ, 647, 922

Egami, E., Rex, M., Rawle, T. D., et al. 2010, A\&A, 518, L12

Ellis, R., Santos, M. R., Kneib, J.-P., \& Kuijken, K. 2001, ApJ, 560, L119

Fazio, G. G., Hora, J. L., Allen, L. E., et al. 2004, ApJS, 154, 10

Finkelstein, S. L., Papovich, C., Giavalisco, M., et al. 2010, ApJ, 719, 1250

Fontana, A., Vanzella, E., Pentericci, L., et al. 2010, ApJ, 725, 205

González, V., Labbé, I., Bouwens, R. J., et al. 2010, ApJ, 713, 115

Hibon, P., Cuby, J.-G., Willis, J., et al. 2010, A\&A, 515, A97

Hickey, S., Bunker, A., Jarvis, M. J., Chiu, K., \& Bonfield, D. 2010, MNRAS, 404, 212

Hu, E. M., Cowie, L. L., McMahon, R. G., et al. 2002, ApJ, 568, L75

Ilbert, O., Tresse, L., Zucca, E., et al. 2005, A\&A, 439, 863

Iye, M., Ota, K., Kashikawa, N., et al. 2006, Nature, 443, 186

Jullo, E., Kneib, J.-P., Limousin, M., et al. 2007, New J. Phys., 9, 447

Kennicutt, R. C. 1998, ARA\&A, 36, 189

Kinney, A. L., Calzetti, D., Bohlin, R. C., et al. 1996, ApJ, 467, 38

Kneib, J. P., Ellis, R. S., Santos, M. R., \& Richard, J. 2004, ApJ, 607, 697

Kodaira, K., Taniguchi, Y., Kashikawa, N., et al. 2003, PASJ, 55, L17

Maizy, A., et al. 2009, A\&A, 509, 105

McLure, R. J., Dunlop, J. S., Cirasuolo, M., et al. 2010, MNRAS, 403, 960

Oesch, P. A., Bouwens, R. J., Carollo, C. M., et al. 2010, ApJ, 709, 21

Ouchi, M., Mobasher, B., Shimasaku, K., et al. 2009, ApJ, 706, 1136

Oke, J. B., \& Gunn, J. E. 1983, ApJ, 266, 713

Persson, S. E., Murphy, D. C., Krzeminski, W., Roth, M., \& Rieke, M. J. 1998, AJ, 116, 2475

Polletta, M., Tajer, M., Maraschi, L., et al. 2007, ApJ, 663, 81

Reddy, N. A., \& Steidel, C. C. 2009, ApJ, 692, 778

Richard, J., Pelló, R., Schaerer, D., Le Borgne, J.-F., \& Kneib, J.-P. 2006, A\&A, 456, 861

Richard, J., Stark, D. P., Ellis, R. S., et al. 2008, ApJ, 685, 705

Rieke, G. H., Young, E. T., Engelbracht, C. W., et al. 2004, ApJS, 154, 25

Rigby, J. R., Marcillac, D., Egami, E., et al. 2008, ApJ, 675, 262

Schaerer, D., \& de Barros, S. 2009, A\&A, 502, 423

Schaerer, D., \& de Barros, S. 2010, A\&A, 515, A73

Schaerer, D., \& Pelló, R. 2005, MNRAS, 362, 1054

Silva, L., Granato, G. L., Bressan, A., \& Danese, L. 1998, ApJ, 509, 103

Trenti, M., \& Stiavelli, M. 2008, ApJ, 676, 767

Vanzella, E., Pentericci, L., Fontana, A., et al. 2011, ApJ, 730, 35

Werner, M. W., Roellig, T. L., Low, F. J., et al. 2004, ApJS, 154, 1

Wiklind, T., Dickinson, M., Ferguson, H. C., et al. 2008, ApJ, 676, 781

Wilkins, S. M., Bunker, A. J., Ellis, R. S., et al. 2010, MNRAS, 403, 938

Zheng, W., Bradley, L. D., Bouwens, R. J., et al. 2009, ApJ, 697, 1907

Zwicky, F. 1937, Phys. Rev. 51, 290 